\title{
VARIATION FORMULAS FOR PRINCIPAL FUNCTIONS, II: APPLICATIONS TO VARIATION FOR HARMONIC SPANS
}

\author{
SACHIKO HAMANO, FUMIO MAITANI, AND HIROSHI \\ YAMAGUCHI
}

\author{
To Professor Mitsuru Nakai, \\ on the occasion of his 77th birthday
}

\begin{abstract}
A domain $D \subset \mathbb{C}_{z}$ admits the circular slit mapping $P(z)$ for $a, b \in$ $D$ such that $P(z)-1 /(z-a)$ is regular at $a$ and $P(b)=0$. We call $p(z)=$ $\log |P(z)|$ the $L_{1}$-principal function and $\alpha=\log \left|P^{\prime}(b)\right|$ the $L_{1}$-constant, and similarly, the radial slit mapping $Q(z)$ implies the $L_{0}$-principal function $q(z)$ and the $L_{0}$-constant $\beta$. We call $s=\alpha-\beta$ the harmonic span for $(D, a, b)$. We show the geometric meaning of $s$. Hamano showed the variation formula for the $L_{1}$-constant $\alpha(t)$ for the moving domain $D(t)$ in $\mathbb{C}_{z}$ with $t \in B:=\{t \in$ $\mathbb{C}:|t|<\rho\}$. We show the corresponding formula for the $L_{0}$-constant $\beta(t)$ for $D(t)$ and combine these to prove that, if the total space $\mathcal{D}=\bigcup_{t \in B}(t, D(t))$ is pseudoconvex in $B \times \mathbb{C}_{z}$, then $s(t)$ is subharmonic on $B$. As a direct application, we have the subharmonicity of $\log \cosh d(t)$ on $B$, where $d(t)$ is the Poincaré distance between $a$ and $b$ on $D(t)$.
\end{abstract}

\section{$\S 1$. Introduction}

Let $R$ be a bordered Riemann surface with boundary $\partial R=C_{1}+\cdots+C_{\nu}$ in a larger Riemann surface $\widetilde{R}$, where $C_{j}$ is a $C^{\omega}$ smooth contour in $\widetilde{R}$. Fix two points $a, b$ with local coordinates $U_{a}:|z|<r_{0}$ and $U_{b}:|z-\xi|<r_{1}$, where $a$ and $b$ correspond to 0 and $\xi$, respectively (where $U_{a}$ and $U_{b}$ have no relations). Among all harmonic functions $u$ on $R \backslash\{a, b\}$ with logarithmic singularity $\log (1 /|z|)$ at $a$ and $\log |z-\xi|$ at $b$ normalized $\lim _{z \rightarrow 0}(u(z)-$ $\log (1 /|z|))=0$, we have two special functions $p$ and $q$ with the boundary conditions that, for each $C_{j}, p$ satisfies $p(z)=$ constant $c_{j}$ on $C_{j}$ and

Received November 5, 2010. Accepted May 12, 2011.

2010 Mathematics Subject Classification. Primary 32T85; Secondary 30C25. 
$\int_{C_{j}} \frac{\partial p(z)}{\partial n_{z}} d s_{z}=0$ (where $\frac{\partial}{\partial n_{z}}$ is the outer normal derivative and $d s_{z}$ is the arc length element at $z$ of $C_{j}$ ), while $q$ satisfies $\frac{\partial q(z)}{\partial n_{z}}=0$ on $C_{j}$. We consider the constant terms $\alpha:=\lim _{z \rightarrow \xi}(p(z)-\log |z-\xi|)$ and $\beta:=\lim _{z \rightarrow \xi}(q(z)-$ $\log |z-\xi|)$. We call $p(z)$ the $L_{1}$-principal function and $\alpha$ the $L_{1}$-constant for $(R, a, b)$ with respect to local coordinates $U_{a}$ and $U_{b}$ or, simply, for $(R, 0, \xi)$, and similarly, we call $q(z)$ the $L_{0}$-principal function and $\beta$ the $L_{0}$-constant (see, [1, Chapter III, Section 3]). Now let $B=\{t \in \mathbb{C}:|t|<\rho\}$, and let $\mathcal{R}: t \in B \rightarrow R(t) \Subset \widetilde{R}$ be a smooth variation of Riemann surfaces $R(t)$ in $\widetilde{R}$ with $t \in B$ such that $\partial R(t)$ is $C^{\omega}$ smooth in $\widetilde{R}$ and $R(t), t \in B$ contains $z=0$ in $U_{a}$ and $\xi(t)$, which vary holomorphically in $U_{b}$. Then each $R(t), t \in B$ admits the $L_{1}$-principal function $p(t, z)$ and $L_{1}$-constant $\alpha(t)$ for $(R(t), 0, \xi(t))$ and, similarly, the $L_{0}$-principal function $q(t, z)$ and the $L_{0}$-constant $\beta(t)$.

Hamano [9] showed the variation formula of the second order for $\alpha(t)$ (see Lemma 2.1 below), which implies that, if the total space $\mathcal{R}=\bigcup_{t \in B}(t, R(t))$ is a pseudoconvex domain in $B \times \widetilde{R}$, then $\alpha(t)$ is subharmonic on $B$. Continuing on [9], we show the variation formula for $\beta(t)$ (see Lemma 2.2 below), which continues on [10]. To prove the formula for $\beta(t)$, we add a new idea to Hamano's proof for $\alpha(t)$. In fact, the formula for $\alpha(t)$ does not concern the genus of $R(t)$, but the formula for $\beta(t)$ does concern it. The formula for $\beta(t)$ implies that, if $\mathcal{R}$ is pseudoconvex in $B \times \widetilde{R}$ and if $R(t), t \in B$ is planar, then $\beta(t)$ is superharmonic on $B$. This contrast between the subharmonicity of $\alpha(t)$ and the superharmonicity of $\beta(t)$ is unified with the notion of the harmonic span $s(t):=\alpha(t)-\beta(t)$ for $(R(t), 0, \xi(t))$ introduced by Nakai (see, [13, Chapter II, Section 3]): if $\mathcal{R}$ is pseudoconvex in $B \times \widetilde{R}$ and $R(t), t \in B$ is planar, then $s(t)$ is subharmonic on $B$; this implies Corollary 4.1. Assume, moreover, that each $R(t), t \in$ is simply connected. Let $\xi_{i}:=\bigcup_{t \in B}\left(t, \xi_{i}(t)\right), i=1,2$ be two holomorphic sections of $\mathcal{R}$ over $B$, and let $d(t)$ denote the Poincaré distance between $\xi_{1}(t)$ and $\xi_{2}(t)$ on $R(t)$. Then $\delta(t):=\log \cosh d(t)$ is subharmonic on $B$. Further, $\delta(t)$ is harmonic on $B$ if and only if $\mathcal{R}$ is fiber-preserving biholomorphic to the product $B \times R(0)$.

\section{$\S 2$. Variation formulas for $L_{0}$-principal functions}

Let $B=\{t \in \mathbb{C}:|t|<\rho\}$, and let $\pi: \widetilde{\mathcal{R}} \rightarrow B$ be a holomorphic family such that $\widetilde{\mathcal{R}}$ is a complex 2 -dimensional manifold, $\pi$ is a holomorphic projection 
from $\widetilde{\mathcal{R}}$ onto $B$, and each fiber $\widetilde{R}(t)=\pi^{-1}(t), t \in B$ is irreducible and nonsingular in $\widetilde{\mathcal{R}}$. We put $\widetilde{\mathcal{R}}=\bigcup_{t \in B}(t, \widetilde{R}(t))$, and we call $\widetilde{R}(t)$ the fiber of $\widetilde{\mathcal{R}}$ over $t \in B$. Let $\mathcal{R}=\bigcup_{t \in B}(t, R(t))$ be a subdomain in $\widetilde{\mathcal{R}}$ such that we have the following conditions:

(1) $\widetilde{R}(t) \ni R(t) \neq \emptyset, t \in B$, and $R(t)$ is a connected Riemann surface of genus $g \geq 0$ such that $\partial R(t)$ in $\widetilde{R}(t)$ consists of a finite number of $C^{\omega}$ smooth contours $C_{j}(t), j=1, \ldots, \nu$;

(2) the boundary $\partial \mathcal{R}=\bigcup_{t \in B}(t, \partial R(t))$ of $\mathcal{R}$ in $\widetilde{\mathcal{R}}$ is $C^{\omega}$ smooth and $\partial \mathcal{R}$ is transverse to each fiber $\widetilde{R}(t), t \in B$.

Note that $g$ and $\nu$ are independent of $t \in B$. Each $C_{j}(t)$ is oriented by $\partial R(t)=C_{1}(t)+\cdots+C_{\nu}(t)$. We regard the complex manifold $\mathcal{R}$ as a variation of Riemann surfaces $R(t)$ with parameter $t \in B$,

$$
\mathcal{R}: t \in B \rightarrow R(t) \Subset \widetilde{R}(t) .
$$

We denote by $\Gamma(B, \mathcal{R})$ the set of all holomorphic sections of $\mathcal{R}$ over $B$. Assume that there exist $\Xi_{0}, \Xi_{\xi} \in \Gamma(B, \mathcal{R})$ with $\Xi_{0} \cap \Xi_{\xi}=\emptyset$ such that there exist $\pi$-local coordinates $U_{0}:=B \times\left\{|z|<r_{0}\right\}$ and $U_{\xi}:=B \times\left\{|z-\xi(t)|<r_{1}\right\}$ of neighborhoods $V_{0}$ of $\Xi_{0}$ and $V_{\xi}$ of $\Xi_{\xi}$ in $\mathcal{R}$ such that $\Xi_{0}$ corresponds to $z=0$ and $\Xi_{\xi}$ corresponds to $z=\xi(t), t \in B$. Let $t \in B$ be fixed. Then $R(t)$ admits the functions $p(t, z)$ and $q(t, z)$ such that both functions are continuous on $\overline{R(t)}$ and harmonic on $R(t) \backslash\{0, \xi(t)\}$ with poles $\log (1 /|z|)$ at $z=0$ and $\log |z-\xi(t)|$ at $z=\xi(t)$ normalized $\lim _{z \rightarrow 0}(p(t, z)-\log (1 /|z|))=$ $\lim _{z \rightarrow 0}(q(t, z)-\log (1 /|z|))=0$ at $z=0$, and $p(t, z)$ and $q(t, z)$ satisfy the following boundary conditions $\left(L_{1}\right)$ and $\left(L_{0}\right)$, respectively: for $j=1, \ldots, \nu$,

$$
\begin{aligned}
& \left(L_{1}\right) \quad p(t, z)=\text { constant } c_{j}(t) \quad \text { on } C_{j}(t) \quad \text { and } \quad \int_{C_{j}(t)} \frac{\partial p(t, z)}{\partial n_{z}} d s_{z}=0 \\
& \left(L_{0}\right) \quad \frac{\partial q(t, z)}{\partial n_{z}}=0 \quad \text { on } C_{j}(t) .
\end{aligned}
$$

We have

$$
\begin{aligned}
& p(t, z)=\log \frac{1}{|z|}+0+h_{0}(t, z) \quad \text { on } U_{0}(t), \\
& q(t, z)=\log \frac{1}{|z|}+0+\mathfrak{h}_{0}(t, z) \quad \text { on } U_{0}(t),
\end{aligned}
$$

where $h_{0}(t, z), \mathfrak{h}_{0}(t, z)$ are harmonic for $z$ on $U_{0}(t)$ such that $h_{0}(t, 0), \mathfrak{h}_{0}(t$, $0) \equiv 0$ on $B$, and 


$$
\begin{aligned}
& p(t, z)=\log |z-\xi(t)|+\alpha(t)+h_{\xi}(t, z) \quad \text { on } U_{\xi}(t), \\
& q(t, z)=\log |z-\xi(t)|+\beta(t)+\mathfrak{h}_{\xi}(t, z) \quad \text { on } U_{\xi}(t),
\end{aligned}
$$

where $\alpha(t), \beta(t)$ are the constant terms and where $h_{\xi}(t, z), \mathfrak{h}_{\xi}(t, z)$ are harmonic for $z$ on $U_{\xi}(t)$ such that $h_{\xi}(t, \xi(t)), \mathfrak{h}_{\xi}(t, \xi(t)) \equiv 0$ on $B$. We call $p(t, z)$ the $L_{1}$-principal function, or simply $L_{1}$-function, and $\alpha$ the $L_{1}$-constant for $(R(t), 0, \xi(t))$, and similarly, we call $q(t, z)$ the $L_{0}$-function and $\beta(t)$ the $L_{0}$ - constant.

The following variation formula is for the second order for $\alpha(t)$.

Lemma 2.1 ([9, Lemma 1.3]). We have

$$
\frac{\partial^{2} \alpha(t)}{\partial t \partial \bar{t}}=\frac{1}{\pi} \int_{\partial R(t)} k_{2}(t, z)\left|\frac{\partial p(t, z)}{\partial z}\right|^{2} d s_{z}+\frac{4}{\pi} \iint_{R(t)}\left|\frac{\partial^{2} p(t, z)}{\partial \bar{t} \partial z}\right|^{2} d x d y
$$

Here

$$
k_{2}(t, z)=\left(\frac{\partial^{2} \varphi}{\partial t \partial \bar{t}}\left|\frac{\partial \varphi}{\partial z}\right|^{2}-2 \Re\left\{\frac{\partial^{2} \varphi}{\partial \bar{t} \partial z} \frac{\partial \varphi}{\partial t} \frac{\partial \varphi}{\partial \bar{z}}\right\}+\left|\frac{\partial \varphi}{\partial t}\right|^{2} \frac{\partial^{2} \varphi}{\partial z \partial \bar{z}}\right) /\left|\frac{\partial \varphi}{\partial z}\right|^{3}
$$

on $\partial \mathcal{R}$, where $\varphi(t, z)$ is a $C^{2}$ defining function of $\partial \mathcal{R}$.

Note that $k_{2}(t, z)$ on $\partial \mathcal{R}$ does not depend on the choice of defining functions $\varphi(t, z)$ of $\partial \mathcal{R}$. We call $k_{2}(t, z)$ the Levi curvature for $\partial \mathcal{R}$ (see $[11,(1.3)]$, $[12,(7)])$.

We give the variation formulas for $\beta(t)$. In the case where $R(t)$ is of genus $g \geq 1$, we need the following consideration, which was not necessary for the variation formulas for $\alpha(t)$. We draw, as usual, $A, B$ cycles $\left\{A_{k}(t), B_{k}(t)\right\}_{1 \leq k \leq g}$ on $R(t)$, which vary continuously in $\mathcal{R}$ with $t \in B$ without passing through $0, \xi(t)$ :

$$
\begin{aligned}
& A_{k}(t) \cap B_{l}(t)=\emptyset \quad \text { for } k \neq l, \quad A_{k} \times B_{k}=1 \quad \text { for } k=1, \ldots, g, \\
& A_{k}(t) \cap A_{l}(t)=B_{k}(t) \cap B_{l}(t)=\emptyset \quad \text { for } k \neq l
\end{aligned}
$$

Here $A_{k}(t) \times B_{k}(t)=1$ means that $A_{k}(t)$ crosses $B_{k}(t)$ once from the righthand side to the left-hand side of the direction $B_{k}(t)$. On $R(t), t \in B$ we put $* d q(t, z)=-\frac{\partial q(t, z)}{\partial y} d x+\frac{\partial q(t, z)}{\partial x} d y$, the conjugate differential of $d q(t, z)$. 
LEMMA 2.2. We have

$$
\begin{aligned}
\frac{\partial^{2} \beta(t)}{\partial t \partial \bar{t}}= & -\frac{1}{\pi} \int_{\partial R(t)} k_{2}(t, z)\left|\frac{\partial q(t, z)}{\partial z}\right|^{2} d s_{z}-\frac{4}{\pi} \iint_{R(t)}\left|\frac{\partial^{2} q(t, z)}{\partial \bar{t} \partial z}\right|^{2} d x d y \\
& -\frac{2}{\pi} \Im \sum_{k=1}^{g}\left(\frac{\partial}{\partial t} \int_{A_{k}(t)} * d q(t, z)\right) \cdot\left(\frac{\partial}{\partial \bar{t}} \int_{B_{k}(t)} * d q(t, z)\right) .
\end{aligned}
$$

Proof. We divide the proof into two steps.

STEP 1. The formula does not depend on the choice of either $\pi$-biholomorphic mappings or $\pi$-local coordinates.

In fact, let $\widetilde{\pi}: \widetilde{\mathcal{D}} \rightarrow B$ be a holomorphic family, and let a subdomain $\mathcal{D}$ of $\widetilde{\mathcal{D}}$ satisfy conditions (1) and (2). We write $\widetilde{\pi}^{-1}(t)=\widetilde{D}(t)$ and $\mathcal{D}=\bigcup_{t \in B}(t, D(t))$, where $D(t) \Subset \widetilde{D}(t)$. Assume that there exists a $\pi$-biholomorphic mapping

$$
T:(t, z) \in \widetilde{\mathcal{R}} \rightarrow(t, w)=(t, F(t, z)) \in \widetilde{\mathcal{D}}
$$

such that $T(\mathcal{R})=\mathcal{D}$. Thus, $R(t)$ and $D(t)$ are equivalent as Riemann surfaces. We write $\widetilde{\Xi}_{0}, \widetilde{\Xi}_{\widetilde{\xi}} \in \Gamma(B, \mathcal{D})$, which correspond to $\Xi_{0}, \Xi_{\xi} \in \Gamma(B, \mathcal{R})$ by $T$. We put $\widetilde{A}_{k}(t)=F\left(t, A_{k}(t)\right)$, and we put $\widetilde{B}_{k}(t)=F\left(t, B_{k}(t)\right)$ on $D(t)$. Since $\int_{A_{k}(t)} * d q(t, z)=\int_{\widetilde{A}_{k}(t)} * d \widetilde{q}(t, w)$, we have

$$
\text { (i) } \frac{\partial}{\partial t} \int_{\widetilde{A}_{k}(t)} * d \widetilde{q}(t, w)=\frac{\partial}{\partial t} \int_{A_{k}(t)} * d q(t, z) \quad \text { for } t \in B,
$$

and similarly for $\widetilde{B}_{k}(t)$ and $B_{k}(t)$. Let $\widetilde{\pi}$-local coordinates $\widetilde{U}_{0}:=B \times\{|w|<$ $\left.\rho_{0}\right\}$ and $\widetilde{U}_{\widetilde{\xi}}:=B \times\left\{|w-\widetilde{\xi}(t)|<\rho_{1}\right\}$ of neighborhoods $\widetilde{V}_{0}$ of $\widetilde{\Xi}_{0}$ and $\widetilde{V}_{\widetilde{\xi}}$ of $\widetilde{\Xi}_{\widetilde{\xi}}$ in $\mathcal{D}$. Each $D(t), t \in B$ admits the $L_{0}$-function $\widetilde{q}(t, w)$ and the $L_{0}$-constant $\widetilde{\beta}(t)$ for $(D(t), 0, \widetilde{\xi})$. We have

$$
\begin{aligned}
& \widetilde{q}(t, w)=\log \frac{1}{|w|}+0+\widetilde{\mathfrak{h}}_{0}(t, w) \quad \text { on }\left\{|w|<\rho_{0}\right\}, \\
& \widetilde{q}(t, w)=\log |w-\widetilde{\xi}(t)|+\widetilde{\beta}(t)+\widetilde{\mathfrak{h}}_{\widetilde{\xi}}(t, w) \quad \text { on }\left\{|w-\widetilde{\xi}(t)|<\rho_{1}\right\},
\end{aligned}
$$

where $\widetilde{\mathfrak{h}}_{0}(t, w)$ is harmonic on $\left\{|w|<\rho_{0}\right\}$ such that $\widetilde{\mathfrak{h}}_{0}(t, 0) \equiv 0$ on $B$, and $\widetilde{h}_{\widetilde{\xi}}(t, z)$ is harmonic on $\left\{|w-\widetilde{\xi}(t)|<\rho_{1}\right\}$ such that $\widetilde{\mathfrak{h}}_{\widetilde{\xi}}(t, \xi(t)) \equiv 0$ on $B$. Then we have the biholomorphic mappings $T_{0}:(t, z) \in U_{0} \rightarrow(t, w)=\left(t, f_{0}(t, z)\right) \in$ $\widetilde{U}_{0}$ such that $f_{0}(t, 0)=0$, and $T_{\xi}:(t, z) \in U_{\xi} \rightarrow(t, w)=\left(t, f_{\xi}(t, z)\right) \in \widetilde{U}_{\widetilde{\xi}}$ such that $f_{\xi}(t, \xi(t))=\widetilde{\xi}(t)$. For $t \in B$, we put $a_{0}(t):=\left.\frac{\partial f_{0}(t, z)}{\partial z}\right|_{z=0}$ and $a_{\xi}(t):=$ 
$\left.\frac{\partial f_{\xi}(t, z)}{\partial z}\right|_{z=\xi(t)}$, so that $a_{0}(t), a_{\xi}(t)$ are nonvanishing holomorphic functions on $B$. We have

$$
\beta(t)=\widetilde{\beta}(t)-\log \left|a_{0}(t)\right|+\log \left|a_{\xi}(t)\right| \quad \text { on } B,
$$

which implies that

$$
\text { (ii) } \frac{\partial^{2} \beta(t)}{\partial t \partial \bar{t}}=\frac{\partial^{2} \widetilde{\beta}(t)}{\partial t \partial \bar{t}} \quad \text { for } t \in B \text {. }
$$

If we write $\widetilde{k}_{2}(t, w)$ for the Levi curvature for $\partial \mathcal{D}$, then we have

$$
\widetilde{k}_{2}(t, w)=k_{2}(t, z)\left|\frac{\partial F(t, z)}{\partial z}\right|
$$

for $w=F(t, z)$ and $(t, z) \in \partial \mathcal{R}$, and hence

$$
\widetilde{k}_{2}(t, w)\left|\frac{\partial \widetilde{q}(t, w)}{\partial w}\right|^{2}|d w|=k_{2}(t, z)\left|\frac{\partial q(t, z)}{\partial z}\right|^{2}|d z|,
$$

which implies that

$$
(i i i) \int_{\partial D(t)} \widetilde{k}_{2}(t, w)\left|\frac{\partial \widetilde{q}}{\partial w}(t, w)\right|^{2} d s_{w}=\int_{\partial R(t)} k_{2}(t, z)\left|\frac{\partial q}{\partial z}(t, z)\right|^{2} d s_{z}
$$

for $t \in B$. Since

$$
(i v) \iint_{D(t)}\left|\frac{\partial^{2} \widetilde{q}}{\partial \bar{t} \partial w}(t, w)\right|^{2} d u d v=\int_{R(t)}\left|\frac{\partial^{2} q}{\partial \bar{t} \partial z}(t, z)\right|^{2} d x d y
$$

for $t \in B$, equations $(i)-(i v)$ imply Step 1 .

STEP 2. Lemma 2.2 is true.

In fact, it suffices to prove the lemma at $t=0$. If necessary, take a smaller disk $B$ of center 0 . Then by the standard use of the immersion theorem for the open Riemann surfaces due to Nishimura [14] (see also [7]), we have a $\pi$ biholomorphic mapping from $\widetilde{\mathcal{R}}$ to an unramified (Riemann) domain $\widetilde{\mathcal{D}}$ over $B \times \mathbb{C}_{w}$ such that, if we write $T(\mathcal{R})=\mathcal{D}$, then the holomorphic sections $\Xi_{0}$ and $\Xi_{\xi}$ of $\mathcal{R}$ over $B$ correspond to the constant sections $\Xi_{0}:=B \times\{w=0\}$ and $\Xi_{1}:=B \times\{w=1\}$ of $\mathcal{D}$ over $B$. By Step 1, it suffices to show the lemma for the unramified domain $\mathcal{D}$ over $B \times \mathbb{C}_{w}$ and the sections $\Xi_{0}, \Xi_{1} \in \Gamma(B, \mathcal{D})$. For the sake of convenience, we use anew the notation $\widetilde{\mathcal{R}}$ and $\mathcal{R}$ for $\widetilde{\mathcal{D}}$ and $\mathcal{D}$. By condition (1), the boundary $\partial \mathcal{R}$ of $\mathcal{R}$ in $\widetilde{\mathcal{R}}$ is $C^{\omega}$ smooth, and 
each $\widetilde{R}(t), t \in B$ is a Riemann surface sheeted over $\mathbb{C}_{z}$ without ramification points. By condition (2), $R(t)$ is a relatively compact subdomain of $\widetilde{R}(t)$ with $C^{\omega}$ smooth boundary $\partial R(t)$ and $R(t) \ni 0,1$. We have $\mathcal{R}=\bigcup_{t \in B}(t, R(t))$ and $\partial \mathcal{R}=\bigcup_{t \in B}(t, \partial R(t))$, which is transverse to each fiber $\widetilde{R}(t)$. Under these situations we find a neighborhood $V=\bigcup_{j=1}^{\nu} V_{j}$ (disjoint union) of $\partial R(0)=\bigcup_{j=1}^{\nu} C_{j}(0)$ such that $(B \times V) \cap\left(\Xi_{0} \cup \Xi_{1}\right)=\emptyset ; V_{j}$ is a thin tubular neighborhood of $C_{j}(0)$ with $V_{j} \supset C_{j}(t)$ for $t \in B$, and $q(t, z)$ is harmonic on $(R(0) \cup V) \backslash\{0,1\}$. We write $\widehat{R}(0):=R(0) \cup V$, so that $q(t, z)$ is defined in the product $B \times \widehat{R}(0)$. Then, $(2.2)$ becomes

$$
q(t, z)=\log |z-1|+\beta(t)+\mathfrak{h}_{1}(t, z) \quad \text { on } U_{1}(t),
$$

where $\mathfrak{h}_{1}(t, 1) \equiv 0$ on $B$. For $t \in B$ we put $u(t, z):=q(t, z)-q(0, z)$ on $\widehat{R}(0) \backslash$ $\{0,1\}$. By putting $u(t, 0)=0$ and $u(t, 1)=\beta(t)-\beta(0), u(t, z)$ is harmonic on $\widehat{R}(0)$.

Let $0<\varepsilon \ll 1$, let $\gamma_{\varepsilon}(0)=\{|z|<\varepsilon\}$, and let $\gamma_{\varepsilon}(1)=\{|z-1|<\varepsilon\}$. Then,

$$
\int_{\partial R(0)-\partial \gamma_{\varepsilon}(0)-\partial \gamma_{\varepsilon}(1)} u(t, z) \frac{\partial q(0, z)}{\partial n_{z}} d s_{z}-q(0, z) \frac{\partial u(t, z)}{\partial n_{z}} d s_{z}=0 .
$$

Letting $\varepsilon \rightarrow 0$, we have from $\frac{\partial q(0, z)}{\partial n_{z}}=0$ on $C_{j}(0), j=1, \ldots, \nu$,

$$
\beta(t)-\beta(0)=\frac{-1}{2 \pi} \sum_{j=1}^{\nu} \int_{C_{j}(0)} q(0, z) \frac{\partial q(t, z)}{\partial n_{z}} d s_{z}=: \frac{-1}{2 \pi} \sum_{j=1}^{\nu} I_{j}(t) .
$$

We take a point $z_{j}^{0}(t)$ on each $C_{j}(t), t \in B$ such that $z_{j}^{0}(t)$ continuously moves in $\partial \mathcal{R}$ with $t \in B$, and we choose a harmonic conjugate function $q_{j}^{*}(t, z)$ of $q(t, z)$ in $V_{j}$ such that $q_{j}^{*}\left(t, z_{j}^{0}(t)\right)=0$. Since $\frac{\partial q(t, z)}{\partial n_{z}}=0$ on $C_{j}(t), q_{j}^{*}(t, z)$ is single valued in $V_{j}$ and

$$
q_{j}^{*}(t, z)=0 \quad \text { for } z \in C_{j}(t) .
$$

Since $d q_{j}^{*}(t, z)=\frac{\partial q(t, z)}{\partial n_{z}} d s_{z}, d q(0, z)=-\frac{\partial q_{j}^{*}(0, z)}{\partial n_{z}} d s_{z}$ along $C_{j}(0)$, we have

$$
\begin{aligned}
I_{j}(t) & =\int_{C_{j}(0)} q(0, z) d q_{j}^{*}(t, z)=\int_{C_{j}(0)} d\left(q(0, z) q_{j}^{*}(t, z)\right)-q_{j}^{*}(t, z) d q(0, z) \\
& =\int_{C_{j}(0)} q_{j}^{*}(t, z) \frac{\partial q_{j}^{*}(0, z)}{\partial n_{z}} d s_{z} .
\end{aligned}
$$


Differentiating both sides by $t$ and $\bar{t}$ at $t=0$, we have

$$
\frac{\partial^{2} I_{j}}{\partial t \partial \bar{t}}(0)=\int_{C_{j}(0)} \frac{\partial^{2} q_{j}^{*}}{\partial t \partial \bar{t}}(0, z) \frac{\partial q_{j}^{*}(0, z)}{\partial n_{z}} d s_{z}
$$

We recall the following.

Proposition $2.1([9,(1.2)])$. Let $u(t, z)$ be a $C^{2}$ function for $(t, z)$ in a neighborhood $\mathcal{V}_{j}=\bigcup_{t \in B}\left(t, V_{j}(t)\right)$ of $\mathcal{C}_{j}=\bigcup_{t \in B}\left(t, C_{j}(t)\right)$ over $B \times \mathbb{C}_{z}$ such that $u(t, z), t \in B$ is harmonic for $z$ in $V_{j}(t)$ and $u(t, z)=$ a certain constant $c_{j}(t)$ on $C_{j}(t)$. Then

$$
\begin{aligned}
\frac{\partial^{2} u}{\partial t \partial \bar{t}} \frac{\partial u}{\partial n_{z}} d s_{z}= & 2 k_{2}(t, z)\left|\frac{\partial u}{\partial z}\right|^{2} d s_{z}+\frac{\partial^{2} c_{j}(t)}{\partial t \partial \bar{t}} \frac{\partial u}{\partial n_{z}} d s_{z} \\
& +4 \Im\left\{\frac{\partial u}{\partial t} \frac{\partial^{2} u}{\partial \bar{t} \partial z} d z\right\}-4 \Im\left\{\frac{\partial c_{j}(t)}{\partial t} \frac{\partial^{2} u}{\partial \bar{t} \partial z} d z\right\} \quad \text { along } C_{j}(t)
\end{aligned}
$$

We apply this for $u(t, z)=q_{j}^{*}(t, z)$ with (2.6) to (2.7) and obtain

$\frac{\partial^{2} I_{j}}{\partial t \partial \bar{t}}(0)=2 \int_{C_{j}(0)} k_{2}(0, z)\left|\frac{\partial q_{j}^{*}(0, z)}{\partial z}\right|^{2} d s_{z}+4 \Im \int_{C_{j}(0)} \frac{\partial q_{j}^{*}}{\partial t}(0, z) \frac{\partial^{2} q_{j}^{*}}{\partial \bar{t} \partial z}(0, z) d z$

We put

$$
\mathbf{a}_{k}(t)=\int_{A_{k}(t)} * d q(t, z), \quad \mathbf{b}_{k}(t)=\int_{B_{k}(t)} * d q(t, z) .
$$

We fix a point $z^{0}(\neq 0,1)$ such that $B \times\left\{z^{0}\right\} \subset \mathcal{R}$. On $R(t), t \in B$ we choose a branch $q^{*}(t, z)$ of a harmonic conjugate function of $q(t, z)$ on $\widehat{R}(0) \backslash\{0,1\}$ such that $q^{*}\left(t, z^{0}\right)=0$. Since $\int_{C_{j}(0)} * d q(t, z)=0$, we have

$$
q^{*}\left(t, z^{\prime}\right)=q^{*}\left(t, z^{\prime \prime}\right) \quad \bmod \left\{2 \pi, \mathbf{a}_{k}(t), \mathbf{b}_{k}(t)(k=1, \ldots, g)\right\}
$$

for any $z^{\prime}, z^{\prime \prime}$ over the same point $z \in \widehat{R}(0) \backslash\{0,1\}$. We also have $q_{j}^{*}(t, z)-$ $q^{*}(t, z)=c_{j}(t)$ on $V_{j}$, where $c_{j}(t)$ is a certain constant for $z \in V_{j}$. It follows that

$$
\begin{aligned}
& \int_{C_{j}(0)} \frac{\partial q_{j}^{*}}{\partial t}(0, z) \frac{\partial^{2} q_{j}^{*}}{\partial \bar{t} \partial z}(0, z) d z \\
& \quad=\int_{C_{j}(0)} \frac{\partial q^{*}}{\partial t}(0, z) \frac{\partial^{2} q^{*}}{\partial \bar{t} \partial z}(0, z) d z+\frac{\partial c_{j}}{\partial t}(0) \int_{C_{j}(0)} \frac{\partial^{2} q^{*}}{\partial \bar{t} \partial z}(0, z) d z
\end{aligned}
$$


The function $f(t, z):=q^{*}(t, z)-i q(t, z)$ belongs to $C^{\omega}\left(B \times V_{j}\right)$, and $f(t, z)$, $t \in B$ is a single-valued holomorphic function in $V_{j}$. Hence,

$$
\int_{C_{j}(0)} \frac{\partial^{2} q^{*}}{\partial \bar{t} \partial z}(0, z) d z=\frac{1}{2}\left[\frac{\partial}{\partial \bar{t}}\left(\int_{C_{j}(0)} f_{z}^{\prime}(t, z) d z\right)\right]_{t=0}=0 .
$$

Therefore,

$$
\begin{aligned}
\frac{\partial^{2} I_{j}}{\partial t \partial \bar{t}}(0)= & 2 \int_{C_{j}(0)} k_{2}(0, z)\left|\frac{\partial q^{*}(0, z)}{\partial z}\right|^{2} d s_{z} \\
& +4 \Im\left\{\int_{C_{j}(0)} \frac{\partial q^{*}}{\partial t}(0, z) \frac{\partial^{2} q^{*}}{\partial \bar{t} \partial z}(0, z) d z\right\}
\end{aligned}
$$

It follows from (2.5) that

$$
\begin{aligned}
\frac{\partial^{2} \beta}{\partial t \partial \bar{t}}(0)= & -\frac{1}{\pi} \int_{\partial R(0)} k_{2}(0, z)\left|\frac{\partial q^{*}(0, z)}{\partial z}\right|^{2} d s_{z} \\
& -\frac{2}{\pi} \Im\left\{\int_{\partial R(0)} \frac{\partial q^{*}}{\partial t}(0, z) \frac{\partial^{2} q^{*}}{\partial \bar{t} \partial z}(0, z) d z\right\} .
\end{aligned}
$$

We divide the proof into two cases.

CASE 1: $R(t)$ is planar (i.e., $g=0$ ). In this case, each $q^{*}(t, z), t \in B$ is determined up to additive constants $\bmod 2 \pi$. By (2.1) and (2.4), $\frac{\partial q^{*}(t, z)}{\partial t}, t \in$ $B$ is a single-valued harmonic function on $\widehat{R}(0)$, and $\frac{\partial^{2} q^{*}(t, z)}{\partial \bar{t} \partial z}, t \in B$ is a single-valued holomorphic function on $\widehat{R}(0)$. Then,

$$
\int_{\partial R(0)} \frac{\partial q^{*}}{\partial t}(0, z) \frac{\partial^{2} q^{*}}{\partial \bar{t} \partial z}(0, z) d z=2 i \iint_{R(0)}\left|\frac{\partial^{2} q^{*}}{\partial \bar{t} \partial z}(0, z)\right|^{2} d x d y .
$$

Therefore,

$$
\frac{\partial^{2} \beta}{\partial t \partial \bar{t}}(0)=-\frac{1}{\pi} \int_{\partial R(0)} k_{2}(0, z)\left|\frac{\partial q(0, z)}{\partial z}\right|^{2} d s_{z}-\frac{4}{\pi} \iint_{R(0)}\left|\frac{\partial^{2} q}{\partial \bar{t} \partial z}(0, z)\right|^{2} d x d y
$$

which is desired.

CASE 2: $R(t)$ is of genus $g \geq 1$. We put $R^{\prime}(0)=R(0) \backslash \bigcup_{k=1}^{g}\left(A_{k}(0) \cup\right.$ $\left.B_{k}(0)\right)$, and we put $\widehat{R}^{\prime}(0)=R^{\prime}(0) \cup V$, so that $R^{\prime}(0)$ and $\widehat{R}^{\prime}(0)$ are planar Riemann surfaces such that

$$
\partial R^{\prime}(0)=\partial R(0)+\sum_{k=1}^{g}\left(A_{k}^{+}(0)+A_{k}^{-}(0)\right)+\sum_{k=1}^{g}\left(B_{k}^{+}(0)+B_{k}^{-}(0)\right) .
$$


Here $A_{k}^{+}(0)$ is of the same direction of $A_{k}(0), A_{k}^{-}(0)$ is of the opposite direction, and $B_{k}^{+}(0)$ and $B_{k}^{-}(0)$ are similar. For $t \in B$, if we restrict the branch $q^{*}(t, z)$ with $q^{*}\left(t, z^{0}\right)=0$ to $R^{\prime}(0) \backslash\{0,1\}$, then $q^{*}\left(t, z^{\prime}\right)=q^{*}\left(t, z^{\prime \prime}\right) \bmod 2 \pi$ for $z^{\prime}, z^{\prime \prime}$ over the same point $z \in \widehat{R}^{\prime}(0)$. Hence, $\frac{\partial q^{*}}{\partial t}(0, z)$ and $\frac{\partial^{2} q^{*}}{\partial \bar{t} \partial z}(0, z)$ are single-valued harmonic functions on $\widehat{R}^{\prime}(0)$, so that

$$
\begin{aligned}
& \int_{\partial R(0)} \frac{\partial q^{*}}{\partial t}(0, z) \frac{\partial^{2} q^{*}}{\partial \bar{t} \partial z}(0, z) d z \\
& =\iint_{R^{\prime}(0)} d\left(\frac{\partial q^{*}}{\partial t}(0, z) \frac{\partial^{2} q^{*}}{\partial \bar{t} \partial z}(0, z) d z\right) \\
& \quad-\sum_{k=1}^{g} \int_{A_{k}^{ \pm}(0)+B_{k}^{ \pm}(0)} \frac{\partial q^{*}}{\partial t}(0, z) \frac{\partial^{2} q^{*}}{\partial \bar{t} \partial z}(0, z) d z \\
& =: J_{1}-J_{2} .
\end{aligned}
$$

Since $\frac{\partial q^{*}}{\partial \bar{t} \partial z}(0, z)$ is holomorphic on $R^{\prime}(0)$, we have

$$
\begin{aligned}
J_{1} & =2 i \iint_{R(0)}\left|\frac{\partial^{2} q}{\partial t \partial \bar{z}}(0, z)\right|^{2} d x d y \\
J_{2}\left(A_{k}\right) & :=\int_{A_{k}^{ \pm}(0)} \frac{\partial q^{*}}{\partial t}(0, z) \frac{\partial^{2} q^{*}}{\partial \bar{t} \partial z}(0, z) d z \\
& =\int_{A_{k}(0)}\left(\frac{\partial q^{*}}{\partial t}\left(0, z^{+}\right)-\frac{\partial q^{*}}{\partial t}\left(0, z^{-}\right)\right) \frac{\partial^{2} q^{*}}{\partial \bar{t} \partial z}(0, z) d z
\end{aligned}
$$

By $(2.3)$ and $\int_{C_{j}(0)} * d q(t, z)=0$, it holds that, for $z^{ \pm}$over any $z \in A_{k}(0)$,

$$
q^{*}\left(t, z^{+}\right)-q^{*}\left(t, z^{-}\right)=\int_{B_{k}(0)} * d q(t, \zeta) \quad \bmod 2 \pi .
$$

Therefore,

$$
\frac{\partial q^{*}}{\partial t}\left(t, z^{+}\right)-\frac{\partial q^{*}}{\partial t}\left(t, z^{-}\right)=\frac{\partial}{\partial t} \int_{B_{k}(0)} * d q(t, \zeta)
$$

independent of $z \in A_{k}(0)$. By $\frac{\partial q^{*}(t, z)}{\partial z} d z=(1 / 2)(* d q(t, z)-i d q(t, z))$,

$$
\begin{aligned}
J_{2}\left(A_{k}\right) & =\left[\frac{\partial}{\partial t}\left(\int_{B_{k}(0)} * d q(t, \zeta)\right)\right]_{t=0} \cdot\left[\frac{\partial}{\partial \bar{t}}\left(\int_{A_{k}(0)} \frac{\partial q^{*}(t, z)}{\partial z} d z\right)\right]_{t=0} \\
& =\frac{1}{2} \frac{\partial \mathbf{b}_{k}}{\partial t}(0) \cdot \frac{\partial \mathbf{a}_{k}}{\partial \bar{t}}(0) .
\end{aligned}
$$


By $B_{k}(0) \times A_{k}(0)=-1$, it similarly holds that $J_{2}\left(B_{k}\right)=-(1 / 2) \frac{\partial \mathbf{a}_{k}}{\partial t}(0)$. $\frac{\partial \mathbf{b}_{k}}{\partial \bar{t}}(0)$, so that $J_{2}\left(A_{k}\right)+J_{2}\left(B_{k}\right)=-i \Im\left\{\frac{\partial \mathbf{a}_{k}}{\partial t}(0) \cdot \frac{\partial \mathbf{b}_{k}}{\partial \bar{t}}(0)\right\}$. Therefore,

$$
\begin{aligned}
& \Im\left\{\int_{\partial R(0)} \frac{\partial q^{*}}{\partial t}(0, z) \frac{\partial^{2} q^{*}}{\partial \bar{t} \partial z}(0, z) d z\right\}=\Im\left\{J_{1}-\sum_{k=1}^{g}\left(J_{2}\left(A_{k}\right)+J_{2}\left(B_{k}\right)\right)\right\} \\
& \quad=2 \iint_{R(0)}\left|\frac{\partial^{2} q}{\partial \bar{t} \partial z}(0, z)\right|^{2} d x d y+\Im\left\{\sum_{k=1}^{g} \frac{\partial \mathbf{a}_{k}}{\partial t}(0) \cdot \frac{\partial \mathbf{b}_{k}}{\partial \bar{t}}(0)\right\} .
\end{aligned}
$$

This completes Step 2.

As noted in [9], since $\mathcal{R}$ is pseudoconvex in $\widetilde{\mathcal{R}}$ if and only if $k_{2}(t, z) \geq 0$ on $\partial \mathcal{R}$, Lemma 2.1 implies that, if $\mathcal{R}$ is pseudoconvex in $\widetilde{\mathcal{R}}$, then the $L_{1^{-}}$ constant $\alpha(t)$ for $(R(t), 0, \xi(t))$ is $C^{\omega}$ subharmonic on $B$, while Lemma 2.2 makes the following contrast with it.

TheOREM 2.1. If $\mathcal{R}$ is pseudoconvex in $\widetilde{\mathcal{R}}$ and $R(t), t \in B$ is planar, then the $L_{0}$-constant $\beta(t)$ for $(R(t), 0, \xi(t))$ is $C^{\omega}$ superharmonic on $B$.

REMARK 2.1. There are examples of $\pi: \mathcal{R} \rightarrow B$ such that $R(t), t \in B$ is not planar and $\beta(t)$ is not superharmonic on $B$.

In fact, let $\pi: \widehat{\mathcal{R}} \rightarrow B$ be a holomorphic family such that $\widehat{R}(t)=\pi^{-1}(t), t \in$ $B$ is a compact Riemann surface of genus $g \geq 1$, and $\widehat{R}(t)$ is irreducible and nonsingular in $\widehat{\mathcal{R}}$ (where $\widehat{\mathcal{R}}$ may be the trivial $B \times \widehat{R}(0)$ ). Let $\Xi_{0}, \Xi_{\xi} \in$ $\Gamma(B, \widehat{\mathcal{R}})$, and use the same notation $U_{0}, U_{\xi}$ as in the proof of Lemma 2.2. The compact Riemann surface $\widehat{R}(t), t \in B$ admits a harmonic function $\widehat{p}(t, z)$ with poles $\log (1 /|z|)$ at $z=0$ and $\log |z-\xi(t)|$ at $z=\xi(t)$ normalized $\lim _{z \rightarrow 0}(\widehat{p}(t, z)-\log (1 /|z|))=0$. We put

$$
\widehat{p}(t, z)=\log |z-\xi(t)|+\widehat{\alpha}(t)+\widehat{h}(t, z) \quad \text { on } U_{\xi}(t),
$$

where $\widehat{h}(t, \xi(t)) \equiv 0$ on $B$. Then $\frac{\partial \widehat{p}(t, z)}{\partial z} d z$ is a meromorphic differential of the third kind on $R(t)$ with poles $-1 / z$ at $z=0$ and $1 /(z-\xi(t))$ at $z=\xi(t)$. If necessary, take a slightly different $\Xi_{\xi} \in \Gamma(B, \widehat{\mathcal{R}})$. Then, since $R(t)$ is of genus $g \geq 1, \frac{\partial \widehat{p}(t, z)}{\partial z} d z$ is not holomorphic for $t \in B$; that is, $\frac{\partial^{2} \widehat{p}(t, z)}{\partial \bar{t} \partial z} d z \not \equiv 0$ on $R(t)$. We choose an $\eta \in \Gamma(B, \widehat{\mathcal{R}})$ with $\eta \cap\left(\Xi_{0} \cup \Xi_{\xi}\right)=\emptyset$ and a $\pi$-local coordinate $U_{\eta}:=B \times\left\{|w|<r_{2}\right\}$ of a neighborhood $V_{\eta}$ of $\eta$ in $\widehat{\mathcal{R}}$ such that $V_{\eta} \cap\left(V_{0} \cup V_{\xi}\right)=\emptyset$. For integer $n \geq 1$ with $1 / n<r_{2}$, we put

$$
\mathcal{R}_{n}=\widehat{\mathcal{R}} \backslash(B \times\{|w| \leq 1 / n\}) .
$$


Then $\pi: \mathcal{R}_{n} \rightarrow B$ is a holomorphic family with conditions (1) and (2). Each $R_{n}(t), t \in B$ admits the $L_{1}$-function $p_{n}(t, z)$ and the $L_{1}$-constant $\alpha_{n}(t)$ for $\left(R_{n}(t), 0, \xi(t)\right)$, and the $L_{0}$-function $q_{n}(t, z)$ and the $L_{0}$-constant $\beta_{n}(t)$. Since the Levi curvature $k_{n 2}(t, z)$ for $\partial \mathcal{R}_{n}=B \times\{|w|=1 / n\}$ vanishes on $\partial \mathcal{R}_{n}$, Lemmas 2.1 and 2.2 reduce to

$$
\begin{aligned}
\frac{\partial^{2} \alpha_{n}(t)}{\partial t \partial \bar{t}}= & \frac{4}{\pi} \iint_{R_{n}(t)}\left|\frac{\partial^{2} p_{n}(t, z)}{\partial \bar{t} \partial z}\right|^{2} d x d y \\
\frac{\partial^{2} \beta_{n}(t)}{\partial t \partial \bar{t}}= & -\frac{4}{\pi} \iint_{R_{n}(t)}\left|\frac{\partial^{2} q_{n}(t, z)}{\partial \bar{t} \partial z}\right|^{2} d x d y \\
& -\frac{2}{\pi} \Im \sum_{k=1}^{q}\left(\frac{\partial}{\partial t} \int_{A_{k}(t)} * d q_{n}(t, z)\right) \cdot\left(\frac{\partial}{\partial \bar{t}} \int_{B_{k}(t)} * d q_{n}(t, z)\right) .
\end{aligned}
$$

It is known (see [1, Chapter III, Section 2]) that, for $t \in B$, both Dirichlet integrals $\| d\left(p_{n}(t, z)-\widehat{p}(t, z) \|_{R_{n}(t)}^{2}\right.$ and $\| d\left(q_{n}(t, z)-\widehat{p}(t, z) \|_{R_{n}(t)}^{2}\right.$ converge to 0 as $n \rightarrow \infty$, so that both $p_{n}(t, z)$ and $q_{n}(t, z)$ locally uniformly converge to $\widehat{p}(t, z)$ in $\widehat{R}(t) \backslash\{0, \xi(t)\}$, and hence both $\alpha_{n}(t)$ and $\beta_{n}(t)$ converge to $\widehat{\alpha}(t)$.

We have

$$
\begin{aligned}
\lim _{n \rightarrow \infty} \frac{\partial^{2} \alpha_{n}(t)}{\partial t \partial \bar{t}} & =\lim _{n \rightarrow \infty} \frac{\partial^{2} \beta_{n}(t)}{\partial t \partial \bar{t}}=\frac{\partial^{2} \widehat{\alpha}(t)}{\partial t \partial \bar{t}} \\
\frac{\partial^{2} \widehat{\alpha}(t)}{\partial t \partial \bar{t}} & =\frac{4}{\pi} \iint_{\widehat{R}(t)}\left|\frac{\partial^{2} \widehat{p}(t, z)}{\partial \bar{t} \partial z}\right|^{2} d x d y \\
& =+\frac{2}{\pi} \Im \sum_{k=1}^{q}\left(\frac{\partial}{\partial t} \int_{A_{k}(t)} * d \widehat{p}(t, z)\right) \cdot\left(\frac{\partial}{\partial \bar{t}} \int_{B_{k}(t)} * d \widehat{p}(t, z)\right)
\end{aligned}
$$

which implies that $\frac{\partial^{2} \widehat{\alpha}(t)}{\partial t \partial \bar{t}}>0$ on $B$ and $\frac{\partial^{2} \beta_{n}(t)}{\partial t \partial \bar{t}}>0$ on $B$ for sufficiently large $n$. Thus, $\pi: \mathcal{R}_{n} \rightarrow B$ is a desired example.

We show the following variation formulas of $\alpha(t)$ and $\beta(t)$ of the firstorder $\frac{\partial \alpha(t)}{\partial t}$ and $\frac{\partial \beta(t)}{\partial t}$ under the same situations for the unramified domain $\mathcal{R}$ over $B \times \mathbb{C}_{z}$ as in Step 2 in the proof of Lemma 2.2 for general $\Xi_{\xi}: t \in$ $B \rightarrow \xi(t) \in R(t)$ instead of $\Xi_{1}:=B \times\{z=1\}$.

LEMMA 2.3. We have

$$
\frac{\partial \alpha(t)}{\partial t}=\frac{1}{\pi} \int_{\partial R(t)} k_{1}(t, z)\left|\frac{\partial p(t, z)}{\partial z}\right|^{2} d s_{z}+\left.2 \frac{\partial h_{\xi}}{\partial z}\right|_{(t, \xi(t))} \cdot \xi^{\prime}(t)
$$




$$
\frac{\partial \beta(t)}{\partial t}=-\frac{1}{\pi} \int_{\partial R(t)} k_{1}(t, z)\left|\frac{\partial q(t, z)}{\partial z}\right|^{2} d s_{z}+\left.2 \frac{\partial \mathfrak{h}_{\xi}}{\partial z}\right|_{(t, \xi(t))} \cdot \xi^{\prime}(t)
$$

Here

$$
k_{1}(t, z)=\frac{\partial \varphi}{\partial t} /\left|\frac{\partial \varphi}{\partial z}\right| \quad \text { on } \partial \mathcal{R}
$$

and $\varphi(t, z)$ is a $C^{2}$ defining function of $\partial \mathcal{R}$.

The function $k_{1}(t, z)$ on $\partial \mathcal{R}$ is due to Hadamard. We note that $k_{1}(t, z)$ on $\partial \mathcal{R}$ as well as $k_{2}(t, z)$ does not depend on the choice of the defining functions $\varphi(t, z)$ for $\partial \mathcal{R}$. Contrary to the cases of $\frac{\partial^{2} \beta(t)}{\partial t \partial \bar{t}}$ and $k_{2}(t, z)\left|\frac{\partial q(t, z)}{\partial z}\right|^{2} d s_{z}, \frac{\partial \alpha(t)}{\partial t}$ and $k_{1}(t, z)\left|\frac{\partial p(t, z)}{\partial z}\right|^{2} d s_{z}$ (and similar to $\beta(t)$ ) depend on the $\pi$-biholomorphic mappings and $\pi$-local coordinates.

Proof. Since the proofs for $\alpha(t)$ and $\beta(t)$ are similar, we give the proof for $\beta(t)$. We divide it into two steps.

STEP 1. Lemma 2.3 is true in the case where $\Xi_{\xi}$ is a constant section on $B$.

In fact, we simply put $\Xi_{\xi}:=B \times\{z=1\}$. Similar to $(2.7)$, we have

$$
\frac{\partial I_{j}}{\partial t}(0)=\int_{C_{j}(0)} \frac{\partial q_{j}^{*}}{\partial t}(0, z) \frac{\partial q_{j}^{*}(0, z)}{\partial n_{z}} d s_{z} .
$$

Under the same notation $u(t, z)$ and $C_{j}(t)$ as in Proposition 2.1, we similarly have

$$
\frac{\partial u}{\partial t} \frac{\partial u}{\partial n_{z}} d s_{z}=2 k_{1}(t, z)\left|\frac{\partial u}{\partial z}\right|^{2} d s_{z}+\frac{\partial c_{j}(t)}{\partial t} \frac{\partial u}{\partial n_{z}} d s_{z} \quad \text { along } C_{j}(t)
$$

We apply this for $u(t, z)=q_{j}^{*}(t, z)$ with $(2.6)$ to $(2.8)$ and obtain

$$
\frac{\partial I_{j}}{\partial t}(0)=2 \int_{C_{j}(0)} k_{1}(0, z)\left|\frac{\partial q_{j}^{*}(0, z)}{\partial z}\right|^{2} d s_{z}
$$

Therefore,

$$
\frac{\partial \beta}{\partial t}(0)=-\frac{1}{\pi} \int_{\partial R(0)} k_{1}(0, z)\left|\frac{\partial q(0, z)}{\partial z}\right|^{2} d s_{z} \quad \text { by }(2.5),
$$

which proves Step 1.

STEP 2. Lemma 2.3 is true for general $\Xi_{\xi}$ on B. 
In fact, it suffices to prove Lemma 2.2 at $t=0$. If necessary, take a smaller disk $B$ of center 0 . Then we find a biholomorphism $T:(t, z) \in B \times \mathbb{P}_{z} \mapsto$ $(t, w)=(t, f(t, z)) \in B \times \mathbb{P}_{w}$ such that $f(t, z)$ is a linear transformation for $z, f(t, 0)=0, \frac{\partial f}{\partial z}(t, 0)=1, f(t, \xi(t))=$ constant $c$ for $t \in B$, and $\mathcal{D}:=T(\mathcal{R})$ is an unramified domain over $B \times \mathbb{C}_{w}$. We write $D(t)=f(t, R(t)), t \in B$, so that $\mathcal{D}=\bigcup_{t \in B}(t, D(t))$ and $\mathcal{D}$ has two constant sections $\Theta_{0}:=B \times\{w=$ $0\}$ and $\Theta_{c}:=B \times\{w=c\}$. Thus, $\mathcal{D}: t \in B \rightarrow D(t)$ is a case in Step 1 . For $t \in B$, we have the $L_{0}$-function $\widetilde{q}(t, w)$ and the $L_{0}$-constant $\widetilde{\beta}(t)$ for $(D(t), 0, c)$, so that

$$
\begin{aligned}
& \widetilde{q}(t, w)=\log \frac{1}{|w|}+\widetilde{\mathfrak{h}}_{0}(t, w) \quad \text { in } U_{0}(t), \\
& \widetilde{q}(t, w)=\log |w-c|+\widetilde{\beta}(t)+\widetilde{\mathfrak{h}}_{c}(t, w) \quad \text { in } U_{c}(t),
\end{aligned}
$$

where $\widetilde{\mathfrak{h}}_{0}(t, 0), \widetilde{\mathfrak{h}}_{c}(t, c) \equiv 0$ on $B$. We put $\widetilde{A}_{k}(t)=f\left(t, A_{k}(t)\right)$ and $\widetilde{B}_{k}(t)=$ $f\left(t, B_{k}(t)\right)$ on $D(t)$, which continuously vary in $\mathcal{D}$ with $t \in B$ without passing through $w=0, c$. Since

$$
w=f(t, z)= \begin{cases}z+b_{2}(t) z^{2}+\cdots & \text { at } z=0 \\ c+a_{1}(t)(z-\xi(t))+a_{2}(t)(z-\xi(t))^{2}+\cdots & \text { at } z=\xi(t)\end{cases}
$$

where $a_{1}(t) \neq 0, a_{2}(t), \ldots, b_{2}(t), \ldots$ are holomorphic on $B$, we have $q(t, z)=$ $\widetilde{q}(t, f(t, z))$ in $\mathcal{R}$; namely,

$$
q(t, z)=\log |f(t, z)-c|+\widetilde{\beta}(t)+\widetilde{\mathfrak{h}}_{c}(t, f(t, z)) \quad \text { at } z=\xi(t)
$$

Therefore,

$$
\begin{aligned}
\beta(t) & =\widetilde{\beta}(t)+\log \left|a_{1}(t)\right|, \\
\mathfrak{h}_{\xi}(t, z) & =\widetilde{\mathfrak{h}}_{c}(t, f(t, z))+\log \left|1+\frac{a_{2}(t)}{a_{1}(t)}(z-\xi(t))+\cdots\right| .
\end{aligned}
$$

Let $\psi(t, w)$ be a $C^{\omega}$ defining function of $\partial \mathcal{D}$. Then $\varphi(t, z):=\psi(t, f(t, z))$ is that of $\partial \mathcal{R}$, so that we have for $w=f(t, z)$

$$
k_{1}(t, z)=\frac{\frac{\partial \varphi(t, z)}{\partial t}}{\left|\frac{\partial \varphi(t, z)}{\partial z}\right|}=\frac{\widetilde{k}_{1}(t, w)}{\left|\frac{\partial f(t, z)}{\partial z}\right|}+\frac{\frac{\partial f(t, z)}{\partial t}}{\left|\frac{\partial f(t, z)}{\partial z}\right|} \cdot \frac{\frac{\partial \psi}{\partial w}(t, w)}{\left|\frac{\partial \psi}{\partial w}(t, w)\right|}, \quad(t, z) \in \partial \mathcal{R} .
$$


Therefore,

$$
\begin{aligned}
& \int_{\partial R(0)} k_{1}(0, z)\left|\frac{\partial q(0, z)}{\partial z}\right|^{2} d s_{z} \\
& =\int_{\partial R(0)} \frac{\widetilde{k}_{1}(0, w)}{\left|\frac{\partial f(0, z)}{\partial z}\right|}\left|\frac{\partial q(0, z)}{\partial z}\right|^{2} d s_{z} \\
& \quad+\int_{\partial R(0)} \frac{\frac{\partial f}{\partial t}(0, z)}{\left|\frac{\partial f(0, z)}{\partial z}\right|} \cdot \frac{\frac{\partial \psi}{\partial w}(0, w)}{\left|\frac{\partial \psi}{\partial w}(0, w)\right|}\left|\frac{\partial q(0, z)}{\partial z}\right|^{2} d s_{z} \\
& =: J_{1}+J_{2} .
\end{aligned}
$$

Since $\frac{\partial \widetilde{q}(0, w)}{\partial w} \frac{f(0, z)}{d z}=\frac{\partial q(0, z)}{\partial z}$, we have, by Step 1 ,

$$
J_{1}=\int_{\partial D(0)} \widetilde{k}_{1}(0, w)\left|\frac{\partial \widetilde{q}(0, w)}{\partial w}\right|^{2} d s_{w}=-\pi \frac{\partial \widetilde{\beta}}{\partial t}(0)=-\pi\left(\frac{\partial \beta}{\partial t}(0)-\frac{1}{2} \frac{a_{1}^{\prime}(0)}{a_{1}(0)}\right)
$$

If we put $z=g(t, w):=f^{-1}(t, w), t \in B ; \widetilde{C}_{j}(0)=f\left(0, C_{j}(0)\right)$; and $\widetilde{V}_{j}=f(0$, $\left.V_{j}\right)$, then we have the single-valued conjugate harmonic function $\widetilde{q}_{j}^{*}(0, w)$ of $\widetilde{q}(0, w)$ in $\widetilde{V}_{j}$ that vanishes on $\widetilde{C}_{j}(0)$, and hence a function $k(w) \in C^{\omega}\left(V_{j}\right)$ such that $\widetilde{q}_{j}^{*}(0, w)=k(w) \psi(0, w)$ in $\widetilde{V}_{j}$, so that

$$
\begin{aligned}
J_{2} & =-\sum_{j=1}^{\nu} \int_{\widetilde{C}_{j}(0)} \frac{\frac{\partial g}{\partial t}(0, w)}{\frac{\partial g(0, w)}{\partial w}} \frac{\frac{\partial \psi(0, w)}{\partial w}}{\left|\frac{\partial \psi(0, w)}{\partial w}\right|}\left|\frac{\partial \widetilde{q}_{j}^{*}(0, w)}{\partial w}\right|^{2} d s_{w} \\
& =i \int_{\partial D(0)} \frac{\frac{\partial g}{\partial t}(0, w)}{\frac{\partial g(0, w)}{\partial w}}\left(\frac{\partial \widetilde{q}^{*}(0, w)}{\partial w}\right)^{2} d w
\end{aligned}
$$

By the residue theorem,

$$
\begin{aligned}
J_{2} & =2 \pi \operatorname{Res}_{w=0, c}\left\{\frac{\frac{\partial g}{\partial t}(0, w)}{\frac{\partial g(0, w)}{\partial w}}\left(\frac{\partial \widetilde{q}(0, w)}{\partial w}\right)^{2}\right\} \\
& =2 \pi\left(\frac{\partial \mathfrak{h}_{\xi}}{\partial z}(0, \xi(0)) \xi^{\prime}(0)-\frac{1}{4} \frac{a_{1}^{\prime}(0)}{a_{1}(0)}\right) .
\end{aligned}
$$

Thus, $J_{1}+J_{2}=-\pi\left(\frac{\partial \beta}{\partial t}(0)-2 \frac{\partial \mathfrak{h}_{\xi}}{\partial z}(0, \xi(0)) \xi^{\prime}(0)\right)$, which is identical with the formula in Lemma 2.3 . 


\section{$\S 3$. Harmonic span and its geometric meaning}

We recall the slit mapping theory in one complex variable. Let $R$ be a planar Riemann surface sheeted over $\mathbb{C}_{z}$ bounded by a finite number of smooth contours $C_{j}, j=1, \ldots, \nu$. Let $a \in R$, and let $U_{a}:=\left\{|z|<r_{0}\right\}$ be a local coordinate of a neighborhood $V_{a}$ of $a$ in $R$. We denote by $\mathcal{U}(R)$ the set of all univalent functions $f$ on $R$ such that $f(z)-1 / z$ is regular at 0 . For $w=f(z) \in \mathcal{U}(R)$ we consider the Euclidean area $E(f)$ of $\mathbb{C}_{w} \backslash f(R)$ and put

$$
\mathcal{E}(R)=\sup \{E(f): f \in \mathcal{U}(R)\} .
$$

Koebe (see [5, Chapter X]) constructed two special $f_{i}(z), i=1,0$ in $\mathcal{U}(R)$ such that $f_{1}(R)$ is a vertical slit domain in $\mathbb{P}_{w}$ and $f_{0}(R)$ is a horizontal slit domain. Grunsky [6, pp. 139-140] considered the function

$$
g:=\frac{1}{2}\left(f_{1}+f_{0}\right) \quad \text { on } R
$$

and showed that each $K_{j}:=-g\left(C_{j}\right), j=1, \ldots, \nu$ bounds an unramified domain $G_{j}$ over $\mathbb{C}_{w}$ such that, if we denote by $E_{j}(g)$ the Euclidean (multivalent) area of $G_{j}$ and put $E(g)=\sum_{j=1}^{\nu} E_{j}(g)$, then $E(g) \geq \mathcal{E}(R)$. Then, Schiffer [16, p. 209] introduced the quantity $S(R)$, called the span for $R$,

$$
S(R):=\Re\left\{a_{1}-b_{1}\right\},
$$

where $a_{1}$ and $b_{1}$ are the coefficients of $z$ (the first degree) of the Taylor expansions of $f_{1}(z)-1 / z$ and $f_{0}(z)-1 / z$ at 0 , respectively, and showed the following beautiful results (see [16, p. 216]): $g \in \mathcal{U}(R)$, each $G_{j}$ is a convex domain in $\mathbb{C}_{w}$, and

$$
E(g)=\mathcal{E}(R)=\frac{\pi}{2} S(R)
$$

His proofs were rather intuitive and short. The precise proofs are found in [1, Chapter III, Section 12].

Let $b \in R, a \neq b$, and let $U_{b}:=\left\{|z-\xi|<r_{1}\right\}$ be a local coordinate of a neighborhood $V_{b}$ of $b$ in $R$. We denote by $\mathcal{S}(R)$ the set of all univalent functions $f$ on $R$ such that $f(z)-1 / z$ is regular at 0 and $f(\xi)=0$, say,

$$
f(z)=c_{1}(z-\xi)+c_{2}(z-\xi)^{2}+\cdots \quad \text { at } \xi
$$

We put $c(f)=c_{1}(\neq 0)$. We draw a simple curve $l$ on $R$ from $\xi$ to 0 . Let $w=f(z) \in \mathcal{S}(R)$. Then $f(l)$ is a simple curve from 0 to $\infty$ in $\mathbb{P}_{w}$, and 
each branch of $\log f(z)$ on $R \backslash l$ is single valued and univalent. Fix one of them, say, $\tau=\log f(z)$. Consider the Euclidean area $E_{\log }(f)(\geq 0)$ of the complement of $\log f(R \backslash l)$ in $\mathbb{C}_{\tau}$, and put

$$
\mathcal{E}_{\log }(R)=\sup \left\{E_{\log }(f): f \in \mathcal{S}(R)\right\} .
$$

Let $p(z)$ and $\alpha$ be the $L_{1}$-function and the $L_{1}$-constant for $(R, 0, \xi)$, and similarly, let $q(z)$ and $\beta$ be the $L_{0}$-function and the $L_{0}$-constant. We choose the harmonic conjugate $p^{*}(z)$ on $R$ such that, if we put $P(z)=e^{p(z)+i p^{*}(z)}$ on $R$, then $P(z)-1 / z$ is regular at 0 . Then $P \in \mathcal{S}(R)$, and $w=P(z)$ is a circular slit mapping with $\log |c(P)|=\alpha$ and $E_{\log }(P)=0$. Similarly, $w=Q(z)=$ $e^{q(z)+i q^{*}(z)}$ is the radial slit mapping with $\log |c(Q)|=\beta$ and $E_{\log }(Q)=0$. We see in [1, Chapter III, Section 4] that $P$ maximizes $2 \pi \log |c(f)|+E_{\log }(f)$, while $Q$ minimizes $2 \pi \log |c(f)|-E_{\log }(f)$ among $\mathcal{S}(R)$.

Nakai (see [13, Chapter II, Section 3]) expected that the quantity

$$
s(R):=\alpha-\beta
$$

will be important as Schiffer span $S(R)$ and named $s(R)$ the harmonic span for $(R, 0, \xi)$. We show that $s(R)$ has some significant properties not only in one complex variable but in the several complex variables.

We write

$$
\begin{aligned}
& P(z)=e^{\alpha+i \theta_{1}}(z-\xi)+\sum_{n=2}^{\infty} a_{n}(z-\xi)^{n} \quad \text { at } \xi \\
& Q(z)=e^{\beta+i \theta_{0}}(z-\xi)+\sum_{n=2}^{\infty} b_{n}(z-\xi)^{n} \quad \text { at } \xi
\end{aligned}
$$

where $\theta_{1}, \theta_{0}$ are certain constants. We put

$$
\begin{aligned}
& D_{1}:=P(R)=\mathbb{P}_{w} \backslash \bigcup_{j=1}^{\nu} P\left(C_{j}\right)=\mathbb{P}_{w} \backslash \bigcup_{j=1}^{\nu} \operatorname{arc}\left\{A_{j}^{(1)}, A_{j}^{(2)}\right\} \\
& D_{0}:=Q(R)=\mathbb{P}_{w} \backslash \bigcup_{j=1} Q\left(C_{j}\right)=\mathbb{P}_{w} \backslash \bigcup_{j=1}^{\nu} \operatorname{segment}\left\{B_{j}^{(1)}, B_{j}^{(2)}\right\} .
\end{aligned}
$$

Here

$$
\begin{aligned}
\operatorname{arc}\left\{A_{j}^{(1)}, A_{j}^{(2)}\right\} & =\left\{r_{j} e^{i \theta}: \theta_{j}^{(1)} \leq \theta \leq \theta_{j}^{(2)}\right\}, \\
\operatorname{segment}\left\{B_{j}^{(1)}, B_{j}^{(2)}\right\} & =\left\{r e^{i \theta_{j}}: 0<r_{j}^{(1)} \leq r \leq r_{j}^{(2)}<\infty\right\},
\end{aligned}
$$

where $0<\theta_{j}^{(2)}-\theta_{j}^{(1)}<2 \pi$ and $r_{j}, \theta_{j}^{(k)}, \theta_{j}, r_{j}^{(k)}(j=1, \ldots, \nu ; k=1,2)$ are con- 
stants. We take the points $a_{j}^{(k)}, b_{j}^{(k)} \in C_{j}$ such that

$$
P\left(a_{j}^{(k)}\right)=A_{j}^{(k)}, \quad Q\left(b_{j}^{(k)}\right)=B_{j}^{(k)} .
$$

Then, $\sqrt{P(z) Q(z)}$ consists of two single-valued branches $H(z)$ and $-H(z)$ on $R$, where $H(z)$ has only one pole at $z=0$ such that $H(z)-1 / z$ is regular at 0 , and $H(z)$ has 0 only at $z=\xi$. We write

$$
H(z)=\sqrt{P(z) Q(z)} \quad \text { on } R
$$

Each branch of $\log P(z)$ and $\log Q(z)$ is also single-valued and univalent on $R \backslash l$, while $\log H(z)$ is single-valued but not univalent so far. We choose three branches in $R \backslash l$ such that

$$
\tau=\log H(z)=\frac{1}{2}(\log P(z)+\log Q(z)) .
$$

We fix a tubular neighborhood $V_{j}$ of each contour $C_{j}$ with $V_{i} \cap V_{j}=\emptyset(i \neq j)$ and $V_{j} \not \supset 0, \xi$, so that $\log H(z)$ on $V_{j}$ is single valued.

Then we have the following geometric meaning of $s(R)$.

THEOREM 3.1. We have the following.

(1) Each $-(\log H)\left(C_{j}\right), j=1, \ldots, \nu$ is a convex curve in $\mathbb{C}_{\tau}$, and $-H\left(C_{j}\right)$ is a simple closed curve in $\mathbb{C}_{w}$.

(2) $H \in \mathcal{S}(R)$, and $E_{\log }(H)=\mathcal{E}_{\log }(R)=(\pi / 2) s(R)$.

(3) Assume that $R$ is simply connected, and let $d(0, \xi)$ denote the Poincaré distance between 0 and $\xi$ on $R$. Then

$$
s(R)=4 \log \cosh d(0, \xi) .
$$

The proofs of Schiffer's results (see [1, Chapter III, Section 12]) do not seem to be available to prove (1) and (2) in Theorem 3.1. We prove them by use of the Schottky double (compact) Riemann surface $\widehat{R}$ of $R$, which is also useful to prove Corollary 4.1 for the variation of Riemann surfaces.

Proof of Theorem 3.1. Similarly to $F:=\frac{d f_{1}}{d f_{0}}$ used in $[1$, p. 182] (see $[16$, (25)]), we consider the function

$$
W=F(z):=\frac{d \log Q}{d \log P}, \quad z \in R \cup \partial R,
$$


which is a single-valued meromorphic function on $R$ such that $\Re F=0$ on $\partial R$, since $\log P\left(C_{j}\right)$ is a vertical segment and $\log Q\left(C_{j}\right)$ is a horizontal segment in $\mathbb{C}_{\tau}$. It follows from the Schwarz reflexion principle that $F$ is meromorphically extended to the Schottky double Riemann surface $\widehat{R}=R \cup \partial R \cup R^{*}$ of $R$ such that $F\left(z^{*}\right)=-\overline{F(z)}$, where $z^{*} \in R^{*}$ is the reflexion point of $z \in R$. Fix $C_{j}, j=1, \ldots, \nu$. Since $\Re \log P(z)=p(z)$ and $\Re \log Q(z)=q(z)$ on $R$, we have

$$
\log P(z)=u_{1}(z)+i v_{1}(z), \quad \log Q(z)=u_{0}(z)+i v_{0}(z), \quad z \in V_{j},
$$

where $u_{1}(z)=$ constant $c_{1}$ and $v_{0}(z)=$ constant $c_{0}$ on $C_{j}$. Then $\mathfrak{C}_{j}:=$ $\log H\left(C_{j}\right)$ is a closed (not necessarily simple so far) curve in $\mathbb{C}_{\tau}$

$$
\tau=\frac{1}{2}\left(c_{1}+u_{0}(z)\right)+\frac{i}{2}\left(c_{0}+v_{1}(z)\right), \quad z \in C_{j} .
$$

Using notation (3.4), we show that

(i) $\left\{a_{j}^{(k)}, b_{j}^{(k)}\right\}_{k=1,2}$ are four distinct points, which necessarily line cyclically, for example, $\left(a_{j}^{(1)}, b_{j}^{(1)}, a_{j}^{(2)}, b_{j}^{(2)}\right)$ on $C_{j}$;

(ii) the zeros of $F(z)$ are $\left\{b_{j}^{(k)}\right\}_{j=1, \ldots, \nu ; k=1,2}$ of order 1 , and the poles are $\left\{a_{j}^{(k)}\right\}_{j=1, \ldots, \nu ; k=1,2}$ of order 1 ;

(iii) the curve $\mathfrak{C}_{j}$ is locally nonsingular in $\mathbb{C}_{\tau}$;

(iv) $\Re F(z)>0$ on $R$;

(v) at any $\tau \in \mathfrak{C}_{j}$, the curvature $1 /\left(\rho_{j}(\tau)\right)$ of $\mathfrak{C}_{j}$ is negative.

We divide the proof into two steps.

SteP 1. If we admit (i), then (ii)-(v) hold.

In fact, (i) clearly implies (iii). Since $P(z)$ is a circular slit mapping on $R$, and $Q(z)$ is a radial slit mapping on $R$, we have $F(z) \neq 0, \infty$ on $R \cup R^{*}$ and $F(z)$ has zeros at most $b_{j}^{(k)}$ and poles at most $a_{j}^{(k)}$, of order 1 . It follows that (i) implies (ii). Further, (i) implies that $W=F(z)$ is locally one-to-one in a neighborhood of at any $z \in C_{j}$ even at $a_{j}^{(k)}, b_{j}^{(k)}(k=1,2)$, so that $F$ is a meromorphic function on $\widehat{R}$ of degree $2 \nu$. Hence, for a fixed $j=1, \ldots, \nu$, if $z$ travels $C_{j}$ all once, then $F(z)$ travels the imaginary axis all just twice. It follows that $F(\widehat{R})$ is a $2 \nu$ sheeted compact Riemann surface over $\mathbb{P}_{W}$ with $2(2 \nu+g-1)$ branch points lying on $\mathbb{P}_{W} \backslash\{\Re W=0\}$, and hence $F(\widehat{R})$ is divided by $\nu$ closed curves $F\left(C_{j}\right)$ into two connected parts over $\Re W>0$ and $\Re W<0$. Since $F(0)=1$, we have $\Re F(z)>0$ on $R$ and $\Re F(z)<0$ on $R^{*}$, which is (iv). To prove (v), fix $p_{0} \in C_{j}$, and take a local parameter 
$z=x+i y$ of a neighborhood $V$ of $p_{0}$ such that $p_{0}$ corresponds to $z=0$ and the oriented arc $C_{j} \cap V$ corresponds to $I:=(-\rho, \rho)$ on the $x$-axis. Using this parameter, we see from $\Re F(z)>0$ on $R$ that

$$
\Im F^{\prime}(x)=\Im \frac{\partial F(x)}{\partial x}<0 \quad \text { on } I .
$$

By (3.7), the subarc $\Gamma_{j}:=\log H(I)$ of $\mathfrak{C}_{j}$ in $\mathbb{C}_{\tau}$ is of the form

$$
\tau=u(x)+i v(x)=\frac{1}{2}\left[\left(c_{1}+u_{0}(x)\right)+i\left(c_{0}+v_{1}(x)\right)\right], \quad x \in I .
$$

Since the $\operatorname{arc} \Gamma_{j}$ is locally nonsingular by (iii), we calculate the curvature $1 / \rho_{j}(x)$ at the point $(u(x), v(x))$ of $\Gamma_{j}$ :

$$
\frac{1}{\rho_{j}(x)}=\frac{v^{\prime \prime}(x) u^{\prime}(x)-v^{\prime}(x) u^{\prime \prime}(x)}{\left(v^{\prime}(x)^{2}+u^{\prime}(x)^{2}\right)^{3 / 2}}=\frac{v_{1}^{\prime \prime}(x) u_{0}^{\prime}(x)-v_{1}^{\prime}(x) u_{0}^{\prime \prime}(x)}{\left(v_{1}^{\prime}(x)^{2}+u_{0}^{\prime}(x)^{2}\right)^{3 / 2}} .
$$

On the other hand, by (3.6) we have, for $x \in I \subset C_{j}$,

$$
\Im F^{\prime}(x)=\Im\left\{\frac{d}{d x}\left(\frac{\frac{d u_{0}(x)}{d x}+i \frac{d c_{0}}{d x}}{\frac{d c_{1}}{d x}+i \frac{d v_{1}(x)}{d x}}\right)\right\}=\frac{v_{1}^{\prime \prime}(x) u_{0}^{\prime}(x)-v_{1}^{\prime}(x) u_{0}^{\prime \prime}(x)}{v_{1}^{\prime}(x)^{2}} .
$$

Therefore,

$$
\frac{1}{\rho_{j}(x)}=\frac{v_{1}^{\prime}(x)^{2}}{\left(v_{1}^{\prime}(x)^{2}+u_{0}^{\prime}(x)^{2}\right)^{3 / 2}} \cdot \Im F^{\prime}(x) .
$$

Since $v_{1}^{\prime}(0)=0$ if and only if $x=a_{j}^{(k)},(3.8)$ proves $(\mathrm{v})$ for $p_{0} \neq a_{j}^{(k)}$. For $p_{0}=a_{j}^{(k)}$, since $v_{1}^{\prime}(0)=0$ and $v_{1}^{\prime \prime}(0), u_{0}^{\prime}(0) \neq 0$ under $(\mathrm{i}), v_{1}^{\prime}(x)^{2} \cdot \Im F^{\prime}(x)$ is regular and $\neq 0$. Hence, $1 / \rho_{j}\left(p_{0}\right)<0$, which proves $(\mathrm{v})$.

STEP 2. Item (i) is true.

In fact, assume that $R$ does not satisfy (i). It does not occur $\left\{a_{j}^{(1)}, a_{j}^{(2)}\right\}=$ $\left\{b_{j}^{(1)}, b_{j}^{(2)}\right\}$ for any $j$, so that $\left\{a_{j}^{(1)}, a_{j}^{(2)}\right\} \cap\left\{b_{j}^{(1)}, b_{j}^{(2)}\right\}$ consists of one point for some $j$, say, $j=1, \ldots, \nu^{\prime}(\leq \nu)$. We denote by $o_{j}$ such a point on $C_{j}$. Hence, each $\mathfrak{C}_{j}:=\log H\left(C_{j}\right), j=1, \ldots, \nu^{\prime}$ is a closed curve in $\mathbb{C}_{\tau}$ with only one singular point at $\mathfrak{o}_{j}:=\log H\left(o_{j}\right)$, and $F$ is a meromorphic function of degree $2 \nu-\nu^{\prime}$ on $\widehat{R}$. By the same reasoning as in Step 1, if $z$ travels $C_{j}, j=1, \ldots, \nu^{\prime}$ all once, then $F(z)$ travels the imaginary axis all just once in $\mathbb{C}_{\tau}$, and $\Re F(z)>0$ on $R$ and $\Re F(z)<0$ on $R^{*}$. This fact implies that 
$1 / \rho_{j}(\tau)<0$ for $\tau \in \mathfrak{C}_{j} \backslash\left\{\mathfrak{o}_{j}\right\}$. To reach a contradiction, we focus to $C_{1}$. We may assume that $\mathfrak{o}_{1}=0$ of $\mathfrak{C}_{1}\left(\subset \mathbb{C}_{\tau}\right)$ and that $a_{1}^{(1)}=b_{1}^{(1)}=o_{1}$ on $C_{1}\left(\subset \mathbb{C}_{z}\right)$. If we take a small subarc $C_{1}^{\prime}$ centered at $o_{1}$ of $C_{1}$ and identify $C_{1}^{\prime}$ with $I=(-r, r)$ on the $x$-axis such that $o_{1}$ corresponds to $0 \in I$, then the subarc $\Gamma:=\log H\left(C_{1}^{\prime}\right)$ of $\mathfrak{C}_{1}$ is written

$$
\tau=\frac{1}{2}\left[\left(a_{2} x^{2}+a_{3} x^{3}+\cdots\right)+i\left(b_{2} x^{2}+b_{3} x^{3}+\cdots\right)\right], \quad x \in I,
$$

where all $a_{k}, b_{k}$ are real and $a_{2}, b_{2} \neq 0$. The other cases being similar, we assume that $a_{2}, b_{2}>0$. We put $\Gamma^{\prime}=\{\log H(x) \in \Gamma: x$ travels from 0 to $r\}$, and similarly, we put $\Gamma^{\prime \prime}$ from 0 to $-r$, so that $\Gamma=-\Gamma^{\prime \prime}+\Gamma^{\prime}$. Since $1 / \rho_{1}(\tau)<$ 0 for $\tau \in \mathfrak{C}_{1} \backslash\left\{\mathfrak{o}_{1}\right\}, \mathfrak{C}_{1}$ has a cusp singularity at $\mathfrak{o}_{1}$ such that $\Gamma^{\prime}$ starts at $\mathfrak{o}_{1}$ whose tangent decreases from $b_{2} / a_{2}>0$ as $x$ travels from 0 to $r$, and similarly for $\Gamma^{\prime \prime}$. We put $\mathfrak{a}=\log H\left(a_{1}^{(2)}\right)$, and we put $\mathfrak{b}=\log H\left(b_{1}^{(2)}\right)$. Since the tangent $T(\tau)$ of $\mathfrak{C}_{1}$ at $\tau=\log H(z)$ is $T(\tau)=v_{1}^{\prime}(z) / u_{0}^{\prime}(z)$, we have $T(\mathfrak{a})=0,|T(\mathfrak{b})|=\infty$ and vice versa. This contradicts that $\mathfrak{C}_{1}$ is a closed curve with $1 / \rho_{1}(\tau)<0$ for any $\tau \in \mathfrak{C}_{1} \backslash\left\{\mathfrak{o}_{1}\right\}$, which proves (i).

The first assertion in Theorem 3.1(1) follows (v). Using notation (3.3), we have

$$
\operatorname{Max}_{z \in C_{j}}\{\Im \log H(z)\}-\operatorname{Min}_{z \in C_{j}}\{\Im \log H(z)\} \leq \frac{1}{2}\left(\theta_{j}^{(2)}-\theta_{j}^{(1)}\right)<\pi .
$$

It follows that the first assertion implies the second assertion in Theorem 3.1(1). To prove (2), given $w^{\prime} \in \mathbb{C}_{w} \backslash \bigcup_{j=1}^{\nu} H\left(C_{j}\right)$, we write $N\left(w^{\prime}\right)$ for the number of $z$ in $R$ such that $H(z)=w^{\prime}$. If we denote by $W_{j}\left(w^{\prime}\right)$ the winding number of $H\left(C_{j}\right)$ about $w^{\prime}$, then we have $W_{j}\left(w^{\prime}\right) \leq 0$ by the second assertion in (1). Since $H(z)$ has only one pole at $z=0$ of order 1 on $R$, we have by the argument principle

$$
N\left(w^{\prime}\right)-1=\sum_{j=1}^{\nu} W_{j}\left(w^{\prime}\right) \leq 0,
$$

so that $N\left(w^{\prime}\right)=0$ or 1 . Hence, $H(z)$ is univalent on $R$, which is the first assertion in (2). To prove the other assertions in (2), let $f \in \mathcal{S}(R)$. We put $u(z):=\log |f(z)|$, and we put $h(z):=\log |H(z)|=(1 / 2)(p(z)+q(z))$. Then $u(z)-h(z)$ is harmonic on the whole $R$, and its Dirichlet integral $D_{R}(u-h):=\|d(u-h)\|_{R}^{2} \geq 0$ is written

$$
D_{R}(u-h)=\int_{\partial R} u d u^{*}-\int_{\partial R} u d h^{*}-\int_{\partial R} h d u^{*}+\int_{\partial R} h d h^{*} .
$$


By $\int_{C_{j}} d u^{*}=0$ and the boundary conditions for $p(z)$ and $q(z)$, we have

$$
\begin{aligned}
\int_{\partial R} u d h^{*} & =\frac{1}{2} \int_{\partial R} u d p^{*}-p d u^{*}=\pi(\log |c(f)|-\alpha), \\
\int_{\partial R} h d u^{*} & =\frac{1}{2} \int_{\partial R} q d u^{*}-u d q^{*}=\pi(\beta-\log |c(f)|) .
\end{aligned}
$$

Therefore,

$$
D_{R}(u-h)=\int_{\partial R} u d u^{*}+\pi(\alpha-\beta)+\int_{\partial R} h d h^{*} .
$$

We put $u=h$, in particular, to obtain $E_{\log }(H)=-\int_{\partial R} h d h^{*}=(\pi / 2)(\alpha-$ $\beta)=(\pi / 2) s(R), E_{\log }(H)-E_{\log }(f)=D_{R}(u-h) \geq 0$, which are desired.

To prove Theorem 3.1(3), we first prove it in the case where $R$ is the disk $D=\{|z|<r\}$ in $\mathbb{C}_{z}$. Let $\xi \in D$. We denote by $p(z)$ and $\alpha$ the $L_{1}$-function and the $L_{1}$-constant for $(D, 0, \xi)$, and similarly for $q(z)$ and $\beta$. We write $P(z)$ and $Q(z)$ the corresponding circular and radial slit mappings on $D$, so that $p(z)=\log |P(z)|$ and $q(z)=\log |Q(z)|$. We have (see [9, Section 5])

$$
\begin{aligned}
P(z) & =\frac{-1}{\xi} \cdot \frac{z-\xi}{z} \cdot\left(1-\frac{z}{r} \frac{\bar{\xi}}{r}\right)^{-1}, \quad z \in D, \\
\alpha & =\log \left|\frac{d P}{d z}(\xi)\right|=-2 \log |\xi|-\log \left(1-\left(\frac{|\xi|}{r}\right)^{2}\right) .
\end{aligned}
$$

Putting $\theta_{\xi}=\arg \xi$, we have

$$
\begin{aligned}
Q(z) & =\frac{1}{r e^{\theta_{\xi}}}\left[\left(\frac{z}{r e^{i \theta_{\xi}}}+\frac{r e^{i \theta_{\xi}}}{z}\right)-\left(\frac{|\xi|}{r}+\frac{r}{|\xi|}\right)\right]=\frac{-1}{\xi} \cdot \frac{z-\xi}{z} \cdot\left(1-\frac{z}{r} \frac{\bar{\xi}}{r}\right) \\
\beta & =\log \left|\frac{d Q}{d z}(\xi)\right|=-2 \log |\xi|+\log \left(1-\left(\frac{|\xi|}{r}\right)^{2}\right) .
\end{aligned}
$$

Hence, the harmonic span $s(D)=\alpha-\beta$ for $(D, 0, \xi)$ is

$$
s(D)=-2 \log \left(1-(|\xi| / r)^{2}\right) \text {. }
$$

Since the Poincaré distance $d(0, \xi)$ between 0 and $\xi$ in $D$ is equal to $(1 / 2) \times$ $\log (1+|\xi| / r) /(1-|\xi| / r)$, we have $s(D)=4 \log \cosh d(0, \xi)$.

For the general $R$, although $\alpha$ and $\beta$ depend on the choice of local coordinates $U_{a}:=\left\{|z|<r_{0}\right\}$ and $U_{b}:=\left\{|w-\xi|<r_{1}\right\}$ about $a$ and $b$, the harmonic span $s(R)=\alpha-\beta$ as well as Poincaré distance does not depend on it. Hence, the first case $R=D$ and the Riemann's mapping theorem imply (3). 
EXAMPLE 3.1. We check Theorem 3.1(1), (2) in the case where $D=\{|z|<$ $r\}$ and $\xi \in D$. By the above formulas,

$$
H(z)=\sqrt{P(z) Q(z)}=1 / z-1 / \xi, \quad z \in D
$$

Thus, $H(z)$ is univalent on $D$. Since $C:=\partial D=\left\{r e^{i \theta}: 0 \leq \theta \leq 2 \pi\right\}$, the closed curve $-H(C)=\left\{\left(e^{i \theta} / r\right)-1 / \xi: 0 \leq \theta \leq 2 \pi\right\}$ is simple and $-\log H(C)$ is a convex curve. Further, we have $E_{\log }(H)=-\pi \log \left(1-|\xi / r|^{2}\right)$. In fact, we prove it for $r=1$ and $|\xi|<1$. Since each branch of $\log (1 / z-1 / \xi)$ is single valued and holomorphic in $\mathbb{C}_{z} \backslash D$, we have by $z \bar{z}=1$ on $C$,

$$
\begin{aligned}
E_{\log }(H) & =\frac{i}{2} \int_{-C} \log (1 / z-1 / \xi) d \overline{\log (1 / z-1 / \xi)} \\
& =\frac{-i}{2} \int_{C} \log (1 / z-1 / \xi) \frac{d z}{z-1 / \bar{\xi}}=-\pi \log \left(1-|\xi|^{2}\right)
\end{aligned}
$$

which is desired. By (3.9), we have $E_{\log }(H)=(\pi / 2) s(D)$.

REMARK 3.1. (1) Let $R_{i}, i=1,2$ be a planar Riemann surface such that $R_{i} \ni 0, \xi$. If we denote by $s_{i}$ the harmonic span for $\left(R_{i}, 0, \xi\right)$, then we have by Theorem $3.1(2)$ that $R_{1} \subset R_{2}$ induces $s_{1} \geq s_{2}$, even when $R_{1}$ and $R_{2}$ are not homeomorphic to each other.

(2) Let $R$ be a planar Riemann surface. As noted in the proof of Theorem $3.1(3)$, the harmonic span $s_{R}(\xi, \eta)$ is a positive function for $(\xi, \eta) \in$ $(R \times R) \backslash \bigcup_{\xi \in R}(\xi, \xi)$. Further, $s_{R}(\xi, \eta)=s_{R}(\eta, \xi)$, and for a fixed $\xi_{0} \in R$, $\lim _{\eta \rightarrow \partial R} s_{R}\left(\xi_{0}, \eta\right)=+\infty$. If we put $s_{R}(\xi, \xi)=0$ for $\xi \in R$, then $s_{R}(\xi, \eta)$ is a $C^{2}$ function on $R \times R$, which satisfies, for a fixed $\xi_{0} \in R$, that there exist $K>0$ and $\delta>0$ such that

$$
\left|\eta-\xi_{0}\right|^{2} / K \leq s\left(\xi_{0}, \eta\right) \leq K\left|\eta-\xi_{0}\right|^{2} \quad \text { for }\left|\eta-\xi_{0}\right|<\delta .
$$

In fact, we may assume that $R$ is a bounded domain in $\mathbb{C}_{z}$ and that $\xi_{0}=0 \in R$. We take $D_{a}:=\{|z|<a\} \Subset R \Subset\{|z|<b\}:=D_{b}$ in $\mathbb{C}_{z}$. By Remark 3.1(1) and (3.9), we have, for $\eta \in D_{a}$,

$$
-\log \left(1-|\eta / b|^{2}\right)=\frac{s_{D_{b}}(0, \eta)}{2} \leq \frac{s_{R}(0, \eta)}{2} \leq \frac{s_{D_{a}}(0, \eta)}{2}=-\log \left(1-|\eta / a|^{2}\right),
$$

which implies (3.10).

We call the function $s_{R}(\xi, \eta)$ on $R \times R$ the $S$-function for $R$. 


\section{§4. Variation formulas for the harmonic spans}

We return to the variation of Riemann surfaces $\mathcal{R}: t \in B \rightarrow R(t)(\Subset \widetilde{R}(t))$ in $\widetilde{\mathcal{R}}=\bigcup_{t \in B}(t, \widetilde{R}(t))$ with conditions (1) and (2) in Section 2. Then Lemmas 2.1 and 2.2 immediately imply the following variation formulas of the harmonic span $s(t)$.

LEMMA 4.1. We have

$$
\begin{aligned}
\frac{\partial s(t)}{\partial t}= & \frac{1}{\pi} \int_{\partial R(t)} k_{1}(t, z)\left(\left|\frac{\partial p(t, z)}{\partial z}\right|^{2}+\left|\frac{\partial q(t, z)}{\partial z}\right|^{2}\right) d s_{z} \\
\frac{\partial^{2} s(t)}{\partial t \partial \bar{t}}= & \frac{1}{\pi} \int_{\partial R(t)} k_{2}(t, z)\left(\left|\frac{\partial p(t, z)}{\partial z}\right|^{2}+\left|\frac{\partial q(t, z)}{\partial z}\right|^{2}\right) d s_{z} \\
& +\frac{4}{\pi} \iint_{R(t)}\left(\left|\frac{\partial^{2} p(t, z)}{\partial \bar{t} \partial z}\right|^{2}+\left|\frac{\partial^{2} q(t, z)}{\partial \bar{t} \partial z}\right|^{2}\right) d x d y \\
& +\frac{2}{\pi} \Im \sum_{k=1}^{g}\left(\frac{\partial}{\partial t} \int_{A_{k}(t)} * d q(t, z)\right) \cdot\left(\frac{\partial}{\partial \bar{t}} \int_{B_{k}(t)} * d q(t, z)\right)
\end{aligned}
$$

We say, in general, that $\mathcal{R}: t \in B \rightarrow R(t)$ is equivalent to a trivial variation if there exists a $\pi$-biholomorphism from the total space $\mathcal{R}$ onto a product space $B \times R_{0}$ (where $R_{0}$ is a Riemann surface).

In the case where $R(t)$ is planar, following (3.2), on $R(t), t \in B$ we have the circular and radial slit mappings

$$
P(t, z)=e^{p(t, z)+i p(t, z)^{*}} \quad \text { and } \quad Q(t, z)=e^{q(t, z)+i q(t, z)^{*}}
$$

such that $P(t, z)-1 / z$ and $Q(t, z)-1 / z$ are regular at $z=0$. We put $D_{1}(t)=$ $P(t, R(t))$, and we put $D_{0}(t)=Q(t, R(t))$, so that

$$
\begin{aligned}
& D_{1}(t)=\mathbb{P}_{w} \backslash \bigcup_{j=1}^{\nu} P\left(t, C_{j}(t)\right)=\mathbb{P}_{w} \backslash \bigcup_{j=1}^{\nu} \operatorname{arc}\left\{A_{j}^{(1)}(t), A_{j}^{(2)}(t)\right\}, \\
& D_{0}(t)=\mathbb{P}_{w} \backslash \bigcup_{j=1} Q\left(t, C_{j}(t)\right)=\mathbb{P}_{w} \backslash \bigcup_{j=1}^{\nu} \operatorname{segment}\left\{B_{j}^{(1)}(t), B_{j}^{(2)}(t)\right\} .
\end{aligned}
$$

TheOrem 4.1. Assume that $\mathcal{R}=\bigcup_{t \in B}(t, R(t))$ is pseudoconvex in $\widetilde{\mathcal{R}}$ and that each $R(t), t \in B$ is planar. Then

(1) $s(t)$ is subharmonic on $B$; 
(2) if $s(t)$ is harmonic on $B$, then

(i) $s(t)$ is constant on $B$, and

(ii) $\mathcal{R}: t \in B \rightarrow R(t)$ is equivalent to a trivial variation. More concretely, $\mathcal{R}$ is $\pi$-biholomorphic to the product domain $B \times \widetilde{D}_{1}$, where $\widetilde{D}_{1}$ is a circular slit domain in $\mathbb{P}_{w}$ such that $\widetilde{D}_{1}=\mathbb{P}_{w} \backslash \bigcup_{j=1}^{\nu}\left\{\widetilde{A}_{j} e^{i \theta}\right.$ : $\left.0 \leq \theta \leq \Theta_{j}\right\}$, where $\widetilde{A}_{1}=1$ and each $\widetilde{A}_{j}(\neq 0), j=2, \ldots, \nu$ is constant, by the holomorphic transformation $T_{0}:(t, z) \in \mathcal{R} \mapsto(t, w)=$ $(t, \widetilde{P}(t, z)) \in B \times \widetilde{D}_{1}$, where $\widetilde{P}(t, z)=P(t, z) / A_{1}^{(1)}(t)$.

Proof. Lemma 4.1 implies (1). To prove (2), we may assume that $\mathcal{R}=$ $\bigcup_{t \in B}(t, R(t))$ is an unramified domain over $B \times \mathbb{C}_{z}$ such that each $R(t), t \in B$ is contained in an unramified planar domain $\widetilde{R}$ over $\mathbb{C}_{z}$, and $\Xi_{0}, \Xi_{\xi}$ are constant sections $B \times\{z=0\}, B \times\{z=1\}$, respectively. Assume that $s(t)$ is harmonic on $B$. By Lemma 4.1, we have

(a) $k_{2}(t, z) \equiv 0$ on $\partial \mathcal{R}$, that is, $\partial \mathcal{R}$ is a Levi flat surface over $B \times \mathbb{C}_{z}$;

(b) both $\frac{\partial p(t, z)}{\partial z}$ and $\frac{\partial q(t, z)}{\partial z}$ are holomorphic for $t \in B$.

By (b) and the normalization at $z=0$, both $w=P(t, z)$ and $w=Q(t, z)$ are holomorphic for two complex variables $(t, z)$ in $\mathcal{R}$ except $B \times\{0\}$. We put $D_{1}(t)=P(t, R(t)) \subset \mathbb{P}_{w}$ for $t \in B$, and $\mathcal{D}_{1}=\bigcup_{t \in B}\left(t, D_{1}(t)\right)$. Since $\mathcal{D}_{1}$ as well as $\mathcal{R}$ over $B \times \mathbb{C}_{z}$ is a pseudoconvex (univalent) domain in $B \times \mathbb{P}_{w}$, it follows from [3, p. 352] that each edge point $A_{j}^{(k)}(t)$ is holomorphic for $t \in B$ and that $A_{j}^{(2)}(t)=A_{j}^{(1)}(t) e^{i \Theta_{j}}$, where $\Theta_{j}$ is constant for $t \in B$. We consider the $\operatorname{map}(t, w) \in \mathcal{D}_{1} \mapsto(t, \widetilde{w})=(t, L(t, w)) \in B \times \mathbb{P}_{\widetilde{w}}$, where $L(t, w)=w / A_{1}^{(1)}(t)$, and we put $\widetilde{\mathcal{D}}_{1}=\bigcup_{t \in B}\left(t, \widetilde{D}_{1}(t)\right)$, where $\widetilde{D}_{1}(t)=L\left(t, D_{1}(t)\right)$. Each $\widetilde{D}_{1}(t), t \in$ $B$ is a circular slit domain in $\mathbb{P}_{\widetilde{w}} \backslash \bigcup_{j=1}^{\nu} \widetilde{C}_{j}(t)$ such that the first circular slit $\widetilde{C}_{1}(t)=\left\{e^{i \theta}: 0 \leq \theta \leq \Theta_{1}\right\}$ is independent of $t \in B$, say, $\widetilde{C}_{1}:=\widetilde{C}_{1}(t)$. Since $\mathcal{R}$ is $\pi$-biholomorphic to $\widetilde{\mathcal{D}}_{1}$, and each $\widetilde{D}_{1}(t), t \in B$ has no ramification points, it suffices for $(2)\left(\right.$ ii) to prove that the edge point $\widetilde{A}_{j}^{(1)}(t):=A_{j}^{(1)}(t) / A_{1}^{(1)}(t)$ of each arc $\widetilde{C}_{j}^{(1)}(t), j=2, \ldots, \nu$ does not depend on $t \in B$.

In fact, we see from (b) that the function $F(t, z)$ defined in (3.5),

$$
W=F(t, z)=\frac{d_{z} \log Q(t, z)}{d_{z} \log P(t, z)}, \quad z \in R(t) \cup \partial R(t),
$$

is holomorphic for $t \in B$ such that $F(t, 0)=1$ and $\Re F(t, z)=0$ on $\partial R(t)$; that is, $F(t, z)$ is a meromorphic function for two complex variables $(t, z) \in$ $\mathcal{R}$ such that $\Re F(t, z)=0$ on $\partial \mathcal{R}$. We put $K_{j}(t)=F\left(t, C_{j}(t)\right)$ in $\mathbb{P}_{W}$. In 
Step 1 of the proof in Theorem 3.1(1) we proved that $K_{j}(t)$ rounds just twice on the imaginary axis in $\mathbb{P}_{W}$. We put $W(t)=F(t, R(t))$, and we put $\mathcal{W}=\bigcup_{t \in B}(t, W(t))$, so that $\partial \mathcal{W}=\bigcup_{t \in B}\left(t, \bigcup_{j=1}^{\nu} K_{j}(t)\right)$, and $\mathcal{R} \approx \mathcal{W}(\pi-$ biholomorphic) by $T:(t, z) \in \mathcal{R} \mapsto(t, W)=(t, F(t, z)) \in \mathcal{W}$. Thus, $W(t)$ has $6 \nu-4$ ramification points. Consider the following $\pi$-biholomorphic mapping $(t, W) \in \mathcal{W} \rightarrow(t, \widetilde{w})=(t, \widetilde{G}(t, W)) \in \widetilde{\mathcal{D}}_{1}$, where $\widetilde{G}(t, W):=L\left(t, P\left(t, F^{-1}(t\right.\right.$, $W))$. We use the following elementary fact.

(*) Let $B=\{|t|<\rho\}$ in $\mathbb{C}_{t}$, and let $E=\{|z|<r\} \cap\{\Re z \geq 0\}$ in $\mathbb{C}_{z}$. If $f(t, z)$ is a holomorphic function for two complex variables $(t, z)$ on $B \times E$ such that $|f(t, z)|=1$ on $B \times(E \cap\{\Re z=0\})$, then $f(t, z)$ does not depend on $t \in B$.

We choose a point $W_{0}$ on $\partial K_{1}(0) \subset \partial \mathcal{W}$ such that $\widetilde{G}\left(0, W_{0}\right)=e^{i \theta_{0}} \in \widetilde{C}_{1}$ with $0<\theta_{0}<\Theta_{1}$ and the direction of $\widetilde{C}_{1}$ at $e^{i \theta_{0}}$ follows as $\theta_{0}$ increases. Then we have a small disk $B_{0} \subset B$ of center 0 and a small half-disk $E=$ $\left\{\left|W-W_{0}\right|<r\right\} \cap\{\Re W \geq 0\}$ in $\mathbb{C}_{W}$ such that $|\widetilde{G}(t, W)| \leq 1$ on $B_{0} \times E$ and $|\widetilde{G}(t, W)|=1$ on $B_{0} \times(E \cap\{\Re W=0\})$. By $(*), \widetilde{G}(t, W)$ for $W \in E \cap\{\Re W \geq$ $0\}$ does not depend on $t \in B_{0}$. By the analytic continuation, $\widetilde{G}(t, W)$ on $\mathcal{W} \cup \partial \mathcal{W}$ does not depend on $t \in B$.

Now assume that some $\widetilde{A}_{j}^{(1)}(t), 2 \leq \exists j \leq \nu$ is not constant for $t \in B$. We take a point $W_{0} \in \mathbb{C}_{W}$ with $\Re W_{0}=0$. Since the component $K_{j}(t)$ of $\partial W(t)$ winds twice around the imaginary axis in $\mathbb{P}_{W}$, for each $t \in B$ we find four points of $K_{j}(t)$ over $W_{0}$. We fix one of them, say, $W_{0}(t) \in K_{j}(t)$, where the corresponding point $z_{j}(t) \in C_{j}(t)$ continuously varies in $\partial \mathcal{R}$ with $t \in B$. Since $\widetilde{C}_{j}(t)=\widetilde{G}\left(t, K_{j}(t)\right)=\left\{\widetilde{A}_{j}^{(1)}(t) e^{i \theta}: 0 \leq \theta \leq \Theta_{j}\right\}$, where $\Theta_{j}$ is constant for $t \in B$, we have $\widetilde{G}\left(t, W_{0}\right)=\widetilde{A}_{j}^{(1)}(t) e^{i \theta(t)}$, where $\theta(t)\left(0<\theta(t)<\Theta_{j}\right)$ continuously varies with $t \in B$. Since $\left|\widetilde{A}_{j}^{(1)}(t)\right|$ as well as $\widetilde{A}_{j}^{(1)}(t)$ is not constant for $t \in B, \widetilde{G}\left(t, W_{0}\right)$ does depend on $t \in B$, a contradiction, and (2)(ii) is proved.

From Remark 3.1(2), the harmonic span $s(t)$ for $(R(t), 0,1)$ is equal to that for $\left(\widetilde{D}_{1}(t), \infty, 0\right)$. Since $\widetilde{D}_{1}(t)=\widetilde{D}_{1}(0)$ for $t \in B, s(t)$ is constant on $B$, which proves $(2)(\mathrm{i})$.

For Theorem 4.1(2)(ii), we cannot replace the condition of the harmonicity of $s(t)$ on $B$ by that of $\alpha(t)$ or $\beta(t)$ on $B$, in general. However, when $R(t), t \in B$ is simply connected, such replacement is possible by the proof of $(2)(\mathrm{ii})$.

Theorem 4.1 and Theorem 3.1(3) directly imply the following. 
Corollary 4.1. Assume that $\mathcal{R}=\bigcup_{t \in B}(t, R(t))$ is pseudoconvex in $\widetilde{\mathcal{R}}$ and that $R(t), t \in B$ is simply connected. Let $\xi_{i} \in \Gamma(B, \mathcal{R}), i=1,2$, and let $d(t)$ denote the Poincaré distance between $\xi_{1}(t)$ and $\xi_{2}(t)$ on $R(t)$. Then $\delta(t):=\log \cosh d(t)$ is subharmonic on $B$. Moreover, $\delta(t)$ is harmonic on $B$ if and only if $\mathcal{R}$ is equivalent to the trivial variation.

Brunella [4, p. 139] said that he could prove the stronger fact, that "Log $d(t)$ is subharmonic on B," using [2] by the same idea.

Corollary 4.2. Assume that $\mathcal{R}=\bigcup_{t \in B}(t, R(t))$ is pseudoconvex in $\widetilde{\mathcal{R}}$ and that each $R(t), t \in B$ is planar. Then the $S$-function $s(t, \xi, \eta)$ for $R(t)$, $t \in B$ is $C^{2}$ plurisubharmonic on $\mathcal{R}^{2}:=\bigcup_{t \in B}(t, R(t) \times R(t))$. In particular, for a fixed $t_{0} \in B$, we simply put $R\left(t_{0}\right)=R$ and $s\left(t_{0}, \xi, \eta\right)=s(\xi, \eta)$. Then $s(\xi, \eta)$ is $C^{2}$ plurisubharmonic on $R \times R$ such that, for any complex line $l$ except $\xi=\eta$ in $R \times R$, the restriction of $s(\xi, \eta)$ on $l \cap(R \times R)$ is strictly subharmonic.

Proof. We may assume that $\widetilde{\mathcal{R}}$ as well as $\mathcal{R}$ is an unramified domain over $B \times \mathbb{C}_{z}$. Let $t \in B \rightarrow(\xi(t), \eta(t)) \in R(t) \times R(t)$ be any holomorphic mapping from $B$ into $\mathcal{R}^{2}$. We put $s(t):=s(t, \xi(t), \eta(t))$ for $t \in B$, and we put $B^{\prime}=B \backslash\{t \in B: \xi(t)=\eta(t)\}$. Consider the translation $T:(t, z) \in \mathcal{R} \mapsto$ $(t, w)=(t, z-\eta(t))$ for $t \in B^{\prime}$, and put $\mathcal{R}_{1}:=T(\mathcal{R})$ and $\xi_{1}=T \xi$. Then $\mathcal{R}_{1}$ is pseudoconvex over $B^{\prime} \times \mathbb{C}_{w}$ and $\xi_{1} \in \Gamma\left(B^{\prime}, \widetilde{\mathcal{R}}\right)$. By Theorem 4.1, the harmonic span $s_{1}(t)$ for $\left(R_{1}(t), 0, \xi_{1}(t)\right)$ is $C^{\omega}$ subharmonic on $B^{\prime}$, and so is $s(t)$ on $B^{\prime}$. It follows from (3.10) that $s(t)$ is $C^{2}$ subharmonic on $B$. By the same argument, we can prove the latter part under the second variation formula in Lemma 4.1 and (3.10). Thus we have the corollary.

In conditions (1) and (2), if we replace $C^{\omega}$ smooth by $C^{\infty}$ smooth, then the results in Sections 2 and 3 hold by replacing $C^{\omega}$ by $C^{\infty}$. In fact, Lemmas 2.1 and 2.2 hold for the $C^{\infty}$ category by not essentially changing the proofs for the $C^{\omega}$ category (see [11, Section 2] and [17, Section 3]).

\section{§5. Approximation theorem for general variations of planar Riemann surfaces}

In this section we consider the general variation of Riemann surfaces $\mathcal{R}: t \in \Delta \rightarrow R(t)$ with the conditions that (a) $\Delta$ is an open or a compact Riemann surface; (b) $\pi: \mathcal{R} \rightarrow \Delta$ is a 2-dimensional holomorphic family such that each fiber $R(t)=\pi^{-1}(t), t \in \Delta$ is irreducible and nonsingular in $\mathcal{R}$; 
(c) each $R(t), t \in B$ is planar; and (d) for every $t \in \Delta$ there exists a neighborhood $B \subset \Delta$ of $t$ such that $\pi^{-1}(B)$ is Stein.

In general, $R(t)$ might be infinite ideal boundary components and $\mathcal{R}: t \in$ $\Delta \rightarrow R(t)$ might not be topologically trivial. For the approximation condition for these variations $\mathcal{R}$, we make the following.

\section{Preparation}

Let $\pi: \mathcal{R} \rightarrow \Delta$ be as above, and let $B \subset \Delta$ be a disk such that $\pi^{-1}(B)$ is Stein. For the sake of convenience we write anew $\Delta:=B$ and $\mathcal{R}:=\pi^{-1}(B)$. Due to Oka-Grauert (see [15, Theorem 8.22]), $\mathcal{R}$ admits a $C^{\omega}$ strictly plurisubharmonic exhaustion function $\psi(t, z)$. Let $\xi: t \in B \rightarrow \xi(t) \in R(t)$ and $\eta: t \in B \rightarrow \eta(t) \in R(t)$ be holomorphic sections of $\mathcal{R}$ over $B$ such that $\xi \cap \eta=\emptyset$. Let $B \Subset \Delta$ be a small disk such that we find a continuous curve $g(t)$ connecting $\xi(t)$ and $\eta(t)$ on $R(t), t \in B$ which continuously varies in $\mathcal{R}$ with $t \in B$. We put $\left.\mathcal{R}\right|_{B}=\bigcup_{t \in B}(t, R(t)) ;\left.\xi\right|_{B}=\bigcup_{t \in B}(t, \xi(t)) ;\left.\eta\right|_{B}=\bigcup_{t \in B}(t, \eta(t))$, and $\left.g\right|_{B}=\bigcup_{t \in B}(t, g(t))$. We take so large $a \gg 1$ that $\left.\mathcal{R}(a)\right|_{B}:=\{(t, z) \in$ $\left.\left.\mathcal{R}\right|_{B}: \psi(t, z)<a\right\}\left.\supset g\right|_{B}$. Then we find a sequence $\left\{a_{n}\right\}_{n}$ with $a_{n}>a$ and $\lim _{n \rightarrow \infty} a_{n}=\infty$ such that

(5.1) $\mathcal{R}_{n}:=$ the connected component of $\left.\mathcal{R}\left(a_{n}\right)\right|_{B}$ that contains $\left.g\right|_{B}$

satisfies (1) each $\mathcal{R}_{n}$ is a connected domain with real 3-dimensional $C^{\omega}$ surfaces $\partial \mathcal{R}_{n}$ in $\left.\mathcal{R}\right|_{B}$ (but each $R_{n}(t), t \in B$ is not always connected); (2) if we consider the set $\mathcal{L}$ of points $t \in B$ such that there exists a point $(t, z(t)) \in \partial \mathcal{R}_{n}$ with $\frac{\partial \psi}{\partial z}(t, z(t))=0$, then $\mathcal{L}$ consists of two kinds of families $\mathcal{L}^{\prime}, \mathcal{L}^{\prime \prime}$ of finite $C^{\omega}$ arcs in $B$

$$
\mathcal{L}^{\prime}=\left\{l_{1}^{\prime}, \ldots, l_{m}^{\prime}\right\}, \quad \mathcal{L}^{\prime \prime}=\left\{l_{1}^{\prime \prime}, \ldots, l_{\mu}^{\prime \prime}\right\}
$$

which have the following property.

For $\mathcal{L}^{\prime}$ : for $t_{0} \in \mathcal{L}^{\prime}$, except a finite set at which some $l_{i}^{\prime}$ and $l_{j}^{\prime}$ or $l_{i}^{\prime}$ itself intersects transversally, say, $t_{0} \in l_{i}^{\prime}, \partial R_{n}\left(t_{0}\right)$ (consisting of a finite number of closed curves) has only one singular point at $z\left(t_{0}\right)$, and we find a bidisk $B_{0} \times V$ of center $\left(t_{0}, z\left(t_{0}\right)\right)$ in $\mathcal{R}_{n+1}$ such that $B_{0} \Subset B$ and $l_{i}^{\prime} \cap B_{0}$ divides $B_{0}$ into two domains $B_{0}^{\prime}$ and $B_{0}^{\prime \prime}$ in the manner that

(i) each $\partial R_{n}(t), t \in B_{0}^{\prime} \cup B_{0}^{\prime \prime}$ has no singular points;

(ii) each $\partial R_{n}(t), t \in l_{i}^{\prime} \cap B_{0}$ has one singular point $z(t)$ at which two subarcs of $\partial R_{n}(t)$ transversally intersect;

(iii) each $R_{n}(t) \cap V, t \in B_{0}^{\prime} \cup\left(l_{i}^{\prime} \cap B_{0}\right)$ consists of two (connected) domains, while each $R_{n}(t) \cap V, t \in B_{0}^{\prime \prime}$ consists of one domain; 
For $\mathcal{L}^{\prime \prime}$ : for $t_{0} \in \mathcal{L}^{\prime \prime}$, except a finite point set, say, $t_{0} \in l_{i}^{\prime \prime}$, we find a unique point $\left(t_{0}, z\left(t_{0}\right)\right) \in \partial \mathcal{R}_{n}$ with $\frac{\partial \psi}{\partial z}\left(t_{0}, z\left(t_{0}\right)\right)=0$, and a bidisk $B_{0} \times V$ of center $\left(t_{0}, z\left(t_{0}\right)\right)$ in $\mathcal{R}_{n+1}$ such that $B_{0} \Subset B$ and $l_{i}^{\prime \prime} \cap B_{0}$ divides $B_{0}$ into two domains $B_{0}^{\prime}$ and $B_{0}^{\prime \prime}$ and $\exists C^{\omega}$ mapping $\mathfrak{z}: t \in l_{i}^{\prime \prime} \cap B_{0} \rightarrow z(t)$ such that $(t, z(t)) \in \partial \mathcal{R}_{n}$ with $\frac{\partial \psi}{\partial z}(t, z(t))=0$ in the manner that

(i) $\left[R_{n}(t) \cup \partial R_{n}(t)\right] \cap V=\emptyset$ for $t \in B_{0}^{\prime} \cup\left(l_{i}^{\prime \prime} \cap B_{0}\right)$;

(ii) $R_{n}(t) \cap V$ for $t \in B_{0}^{\prime \prime}$ is a simply connected domain $\delta_{n}(t)$ such that, for a given $t^{0} \in l_{i}^{\prime \prime} \cap B_{0}, \delta_{n}(t)$ shrinkingly approaches the point $z\left(t^{0}\right)$ as $t \in B_{0}^{\prime \prime} \rightarrow t^{0}$.

For the singular point $z(t), t \in l_{i}^{\prime} \subset \mathcal{L}^{\prime}$, we have the connected component $C(t)$ of $\partial R_{n}(t)$ passing through $z(t)$. Then $C(t)$ consists of one closed curve, or two closed curves $C_{i}(t), i=1,2$, such that $C(t)=C_{1}(t) \cup C_{2}(t)$ and $C_{1}(t) \cap$ $C_{2}(t)=z(t)$. For example, in (FIII) below, $C(t)$ consists of one closed curve, and in (FI) and (FII), $C(t)$ consists of two closed curves.

For the singular $z(t), t \in l_{i}^{\prime \prime} \subset \mathcal{L}^{\prime \prime},(t, z(t)) \in \partial \mathcal{R}_{n}$ but $z(t) \notin \partial R_{n}(t)$.

Fix $t \in B$ and $n \geq 1$, and consider the connected component $R_{n}^{\prime}(t)$ of $R_{n}(t)$ that contains $g(t)$. We put $\mathcal{R}_{n}^{\prime}=\bigcup_{t \in B}\left(t, R_{n}^{\prime}(t)\right)$, and we put $\partial \mathcal{R}_{n}^{\prime}=$ $\bigcup_{t \in B}\left(t, \partial R_{n}^{\prime}(t)\right)$. The variation

$$
\mathcal{R}_{n}^{\prime}: t \in B \rightarrow R_{n}^{\prime}(t)
$$

is no longer a smooth variation of $R_{n}^{\prime}(t)$ with $t \in B$; that is, $\mathcal{R}_{n}^{\prime}$ satisfies neither corresponding condition (1) nor (2) of $\mathcal{R}$ in Section 2. Since $R(t)$ is irreducible in $\mathcal{R}$, we have $R_{n}^{\prime}(t) \Subset R_{n+1}^{\prime}(t), \lim _{n \rightarrow \infty} \mathcal{R}_{n}^{\prime}=\left.\mathcal{R}\right|_{B}$, and $\lim _{n \rightarrow \infty} R_{n}^{\prime}(t)=R(t)$ for $t \in B$. By (i) and (ii) for $\mathcal{L}^{\prime \prime}$, there exists a neighborhood $\mathcal{V}$ of $\bigcup_{t \in \mathcal{L}^{\prime \prime}}(t, z(t))$ in $\mathcal{R}_{n+1}$ such that $\left[\mathcal{R}_{n}^{\prime} \cup \partial \mathcal{R}_{n}^{\prime}\right] \cap \mathcal{V}=\emptyset$, so that $\mathcal{L}^{\prime \prime}$ does not give any influence for the variation $\mathcal{R}_{n}^{\prime}$ (contrary to that for the variation $\mathcal{R}_{n}$ ). Each $R(t), t \in \Delta$ is assumed to be planar. We separate the singular point $z(t)$ of $\partial R_{n}(t), t \in l_{i}^{\prime} \subset \mathcal{L}^{\prime}$ such that $z(t) \in \partial R_{n}^{\prime}(t)$ into the following two cases: let $C(t)$ denote the connected component of $\partial R_{n}(t)$ passing through $z(t)$; then

(c1) $C(t)$ consists of two closed curves $C_{i}(t), i=1,2$, and one of them, say, $C_{1}(t)$, is one of the boundary components of $R_{n}^{\prime}(t)$, so that $\left(C_{2}(t) \backslash\right.$ $\{z(t)\}) \cap \partial R_{n}^{\prime}(t)=\emptyset$

(c2) $C(t)$ is one of the boundary components of $R_{n}^{\prime}(t)$, so that two distinct points of $\partial R_{n}^{\prime}(t)$ lie over $z(t)$.

For example, if the shadowed part below is $R_{n}^{\prime}(t)$, then the singular point $z(t)$ is of case (c1) for (FI), and of case (c2) for (FII) or (FIII). 
(FI)

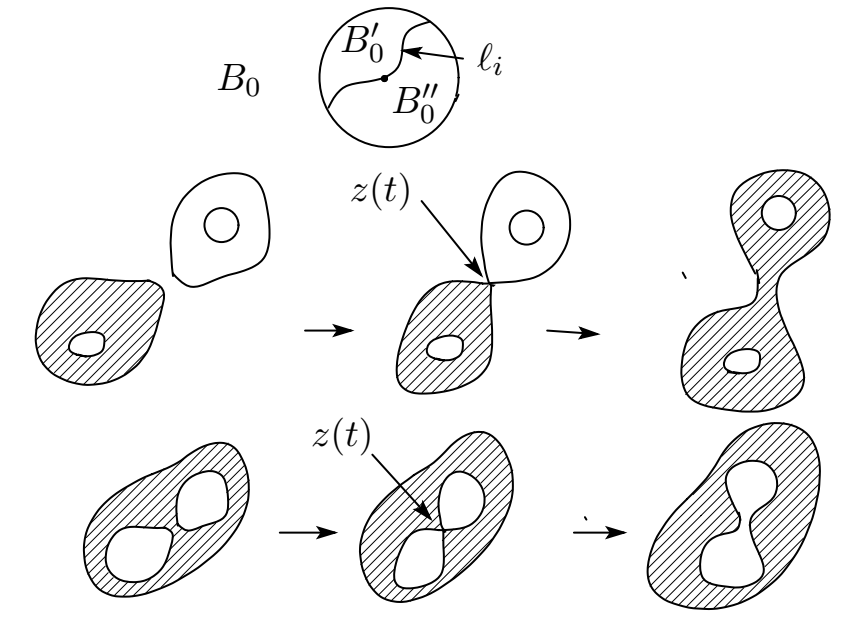

(FII)

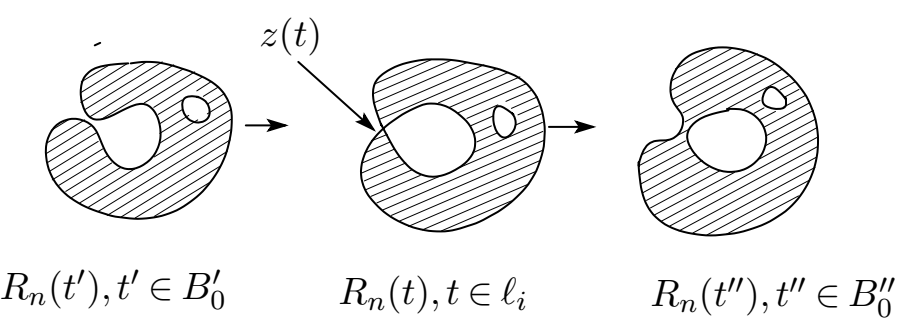

Figure 1: Variation $\mathcal{R}_{n}^{\prime}: t \in B_{0} \rightarrow R_{n}^{\prime}(t)$

For $t \in B$ we consider the $L_{1}$-function $p_{n}(t, z)$, the $L_{0}$-function $q_{n}(t, z)$, and the harmonic span $s_{n}(t)$ for $\left(R_{n}^{\prime}(t), \xi(t), \eta(t)\right)$.

Lemma 5.1 (Hamano [8]). Let $\mathcal{R}$ be a Stein manifold, and each $R(t), t \in \Delta$ is planar. Then we have the following.

(1) $p_{n}(t, z)$ and $q_{n}(t, z)$ are continuous for $(t, z)$ in $\mathcal{R}_{n}^{\prime}$, and $s_{n}(t)$ is continuous on $B$.

(2) Assume that at each singular point $z(t)$ of $\partial R_{n}(t), t \in l_{i}^{\prime} \subset \mathcal{L}^{\prime}$ such that $z(t) \in \partial R_{n}^{\prime}(t)$, case (c1) only occurs. Then

(i) $p_{n}(t, z)$ and $q_{n}(t, z)$ are of class $C^{1}$ for $(t, z)$ on $\mathcal{R}_{n}^{\prime} \backslash\{\xi, \eta\}$;

(ii) $s_{n}(t)$ is $C^{1}$ subharmonic on $B$.

(3) In general, (2) does not hold in case (c2).

As an example of $(\mathrm{FI})$ of $\mathcal{L}^{\prime}$, let $B=\{|t|<1 / 10\}$, let $D=\{|z|<2\}$, let $\psi_{1}=\left(e^{-100+|t|^{2}} /|z-1|^{2}\right)-1$, let $\psi_{2}=\left|z^{2}-1\right|-\left(1-2 \Re t-|t|^{2}\right)$, let $\psi_{3}=$ 
$\left(e^{-100+|t|^{2}} /|z+1|^{2}\right)-1$, and let $\mathcal{R}=\left\{(t, z) \in B \times D: \psi_{1}<0, \psi_{2}<0, \psi_{3}<0\right\}$. Then $\mathcal{R}$ is pseudoconvex in $B \times D$, and the arc $l^{\prime}=\left\{t \in B: 2 \Re t+|t|^{2}=0\right\}$ divides $B$ into two domains $B^{\prime} \cup B^{\prime \prime}$ such that $\partial R(t), t \in l^{\prime}$ consists of two circles $\psi_{1}(t, z)=0, \psi_{3}(t, z)=0$ and the leminiscate $C:\left|z^{2}-1\right|=1$ with singular point $z(t)=0$. We similarly have examples $(\mathrm{FII}),(\mathrm{FIII})$ of $\mathcal{L}^{\prime}$.

As an example of $\mathcal{L}^{\prime \prime}$, let $B, D$ be the same as above. Let $\psi(t, z):=\mid z-$ $\left.t\right|^{2}+|t|^{2}+2 \Re t$, and put $\mathcal{R}=\{(t, z) \in B \times D: \psi(t, z)<0\}$. Then the arc $l^{\prime \prime}=\{t \in B: \phi(t)=0\}$, where $\phi(t)=-|t|^{2}-2 \Re t$, divides $B$ into two domains $B^{\prime}=\{t \in B: \phi(t)<0\}$ and $B^{\prime \prime}=\{t \in B: \phi(t)>0\}$ such that $\frac{\partial \psi}{\partial z}(t, t)=0$ for $t \in l^{\prime \prime}, R(t)=\emptyset$ for $t \in B^{\prime} \cup l^{\prime \prime}$ and $R(t)=\left\{|z-t|^{2}<\phi(t)\right\}$ for $t \in B^{\prime \prime}$. The mapping $\mathfrak{z}: t \in l^{\prime \prime} \rightarrow z(t)=t$ so that $(t, t) \in \partial \mathcal{R}$ but $t \notin \partial R(t)$, and each $R(t), t \in B^{\prime \prime}$ is a disk $\{|z-t|<\phi(t)\}$ that shrinkingly approaches the singular point $z=t^{0}$ as $t \rightarrow t^{0} \in l^{\prime \prime}$.

Since the Stein manifold carries a $C^{\omega}$ strictly plurisubharmonic exhaustion function, we immediately have the following.

Lemma 5.2. Let $\mathcal{R}: t \in \Delta \rightarrow R(t)$ satisfy $(a)-(d)$. Let $\xi, \eta \in \Gamma(\Delta, \mathcal{R})$ such that $\xi \cap \eta=\emptyset$. Assume that

( $\star$ ) $R(t), t \in \Delta$ is homeomorphic to a domain in $\mathbb{C}_{w}$ bounded by a finite number, say, $\nu$, of contours, where $\nu$ is independent of $t \in \Delta$.

Then, for $t_{0} \in \Delta$, there exists a disk $B \Subset \Delta$ of center $t_{0}$ such that we find an increasing sequence $\left\{\mathcal{R}_{n}^{\prime}\right\}_{n}$ of case (c1) with $\lim _{n \rightarrow \infty} \mathcal{R}_{n}^{\prime}=\left.\mathcal{R}\right|_{B}$.

Let $\mathcal{R}: t \in \Delta \rightarrow R(t)$ satisfy (a)-(d), and let $\xi, \eta \in \Gamma(\Delta, \mathcal{R})$ such that $\xi \cap$ $\eta=\emptyset$. We fix a small disk $B \Subset \Delta$ so that we can fix local parameters $(t, z)$ of $\left.\xi\right|_{B}$ and $\left.\eta\right|_{B}$ in $\left.\mathcal{R}\right|_{B}$ and so that $\left\{\mathcal{R}_{n}\right\}_{n}$ satisfies conditions in "Preparation" to these $\Delta$ and $B$. Precisely, we define

(5.2) $\mathcal{R}_{n}:=$ the connected component of $\left.\mathcal{R}\left(a_{n}\right)\right|_{B}$ that contains $\left.g\right|_{B}$,

which satisfies cases (1) and (2) for (5.1). We put $\mathcal{R}_{n}=\bigcup_{t \in B}\left(t, R_{n}(t)\right)$, and for $t \in B$ we denote by $R_{n}^{\prime}(t)$ the connected component of $R_{n}(t)$ that contains $g(t)$ (connecting $\xi(t)$ and $\eta(t))$ and put $\mathcal{R}_{n}^{\prime}=\bigcup_{t \in B}\left(t, R_{n}^{\prime}(t)\right)$. Though $\partial R^{\prime}(t)$ may not be smooth, each $R_{n}^{\prime}(t)$ admits the $L_{1}$-function $p_{n}(t, z)$ and the $L_{1}$-constant $\alpha_{n}$ for $\left(R_{n}^{\prime}(t), 0, \eta(t)\right)$, where $B \times\left\{|z|<r_{1}\right\}$ and $\bigcup_{t \in B}(t$, $\left\{|z-\eta(t)|<r_{2}\right)$ are $\pi$-local coordinates for $\xi$ and $\eta$, and similarly for $q_{n}(t, z)$ and $\beta_{n}(t)$. In one complex variable it is known (see [1, Chapter III, Section 8]) that $p_{n}(t, z)$ uniformly converges to a certain function $p(t, z)$ on any compact set in $R(t) \backslash\{\xi(t), \eta(t)\}$. Thus, $p(t, z)$ is harmonic on $R(t) \backslash$ $\{\xi(t), \eta(t)\}$ with the same pole as $p_{n}(t, z)$ at $\xi(t)$ and $\eta(t)$. Putting $\alpha(t)=$ 
$\lim _{z \rightarrow \eta(t)}(p(t, z)-\log |z-\eta(t)|)$, we have $\lim _{n \rightarrow \infty} \alpha_{n}(t)=\alpha(t)$. We also call $p(t, z)$ and $\alpha(t)$ the $L_{1}$-function and the $L_{1}$-constant for $(R(t), 0, \eta(t))$. Similarly, we define the $L_{0}$-function $q(t, z)$ and the $L_{0}$-constant $\beta(t)$, and call $s(t):=\alpha(t)-\beta(t)$ the harmonic span for $(R(t), \xi(t), \eta(t))$. Since $R(t)$ is planar, we have $s_{n}(t) \searrow s(t)$ as $n \rightarrow \infty$. Their proofs in [1] imply that, for $\left.K \Subset \mathcal{R}\right|_{B} \backslash\left\{\left.\xi\right|_{B},\left.\eta\right|_{B}\right\}$,

$$
p_{n}(t, z), q_{n}(t, z), p(t, z), q(t, z) \text { are uniformly bounded on } K \text {. }
$$

Though $p(t, z), q(t, z), \alpha(t), \beta(t)$ depend on the choice of local coordinates about $\xi(t)$ and $\eta(t), s(t)$ does not depend on it, so that $s(t)(\geq 0)$ is a function on $B$ and on $\Delta$.

Using this notation, we have the following approximation condition.

THEOREM 5.1. Let $\mathcal{R}: t \in \Delta \rightarrow R(t)$ satisfy $(b)-(d)$, where $\Delta$ is an open Riemann surface. Let $\xi, \eta \in \Gamma(\Delta, \mathcal{R})$ such that $\xi \cap \eta=\emptyset$, and let $s(t)$ denote the harmonic span for $(R(t), \xi(t), \eta(t))$. Assume that

(*) for any $t_{0} \in \Delta$, there exists a small disk $B \Subset \Delta$ of center $t_{0}$ such that we find an increasing sequence $\left\{\mathcal{R}_{n}^{\prime}\right\}_{n}$ of case (c1) such that $\lim _{n \rightarrow \infty} \mathcal{R}_{n}^{\prime}=$ $\left.\mathcal{R}\right|_{B}$.

Then

(1) $s(t)$ is subharmonic on $\Delta$;

(2) (simultaneous uniformization) if $s(t)$ is harmonic on $\Delta$, then $\mathcal{R}$ is $\pi$ biholomorphic to a univalent domain in $\Delta \times \mathbb{P}$.

Proof. To show (1), let $t_{0} \in \Delta$. Then we have a disk $B \subset \Delta$ with condition (*). By Lemma $5.1(2)(\mathrm{ii}), s_{n}(t)$ is $C^{1}$ subharmonic on $B$; hence, $s(t)$ is subharmonic on $B$ and on $\Delta$. To prove (2), we cover $\Delta$ by small disks $\left\{B_{i}\right\}_{i=1,2, \ldots}$ with condition $(*)$; that is, for fixed $B_{i}$, we find an increasing sequence $\left\{\mathcal{R}_{n}^{\prime}\right\}_{n}$ (depending on $B_{i}$ ) such that each $\mathcal{R}_{n}^{\prime}$ is of case (c1) and $\lim _{n \rightarrow \infty} \mathcal{R}_{n}^{\prime}=\left.\mathcal{R}\right|_{B_{i}}$. We divide the proof into two steps.

STEP 1. Each $\left.\mathcal{R}\right|_{B_{i}}, i=1,2, \ldots$, is $\pi$-biholomorphic to a univalent domain $\mathcal{D}_{i}$ in $B \times \mathbb{P}$.

In fact, we simply write $B=B_{i}$. We put $\mathcal{R}_{n}^{\prime}=\bigcup_{t \in B}\left(t, R_{n}^{\prime}(t)\right), n=1,2, \ldots$, and consider $p_{n}(t, z), q_{n}(t, z)$ and $s_{n}(t)$ for each $\left(R_{n}^{\prime}(t), 0, \eta(t)\right), t \in B$ as above. We put

$$
\begin{array}{ll}
P_{n}(t, z)=e^{p_{n}(t, z)+i p_{n}(t, z)^{*}}, & P(t, z)=e^{p(t, z)+i p(t, z)^{*}}, \\
Q_{n}(t, z)=e^{q_{n}(t, z)+i q_{n}(t, z)^{*}}, & Q(t, z)=e^{q(t, z)+i q(t, z)^{*}},
\end{array}
$$


which are all 0 at $z=\eta(t)$ and normalized

$$
\frac{1}{z}+\text { (holomorphic function) near } z=0 .
$$

For $t \in B, P_{n}(t, z)$ and $Q_{n}(t, z)$ uniformly converge to $P(t, z)$ and $Q(t, z)$ on any compact set in $R(t) ; w=P_{n}(t, z)$ is a circular slit mapping on $R_{n}^{\prime}(t)$, and similarly $w=Q_{n}(t, z)$ is a radial slit one. Hence, $P(t, z)$ and $Q(t, z)$ are univalent functions on $R(t)$. We also call $P(t, z)$ the circular slit mapping for $(R(t), 0, \eta(t))$, and similarly, we call $Q(t, z)$ the radial slit mapping. For Step 1 it suffices to show that

(a) the harmonicity of $s(t)$ on $B$ implies that $P(t, z)$ is holomorphic for two complex variables $(t, z)$ in $\left.\mathcal{R}\right|_{B} \backslash\left\{\left.\xi\right|_{B}\right\}$.

In fact, fix a point $\left(t_{0}, z_{0}\right)$ in $\left.\mathcal{R}\right|_{B} \backslash\left\{\left.\xi\right|_{B},\left.\eta\right|_{B}\right\}$, and let $B_{0} \times\left. V \Subset \mathcal{R}\right|_{B} \backslash$ $\left\{\left.\xi\right|_{B},\left.\eta\right|_{B}\right\}$ be a bidisk centered at $\left(t_{0}, z_{0}\right)$, a local coordinate of a neighborhood of $\left(t_{0}, z_{0}\right)$. We put $f(t, z):=\frac{\partial p(t, z)}{\partial z}$ for $(t, z) \in B_{0} \times V$. From (5.5) it suffices for $(a)$ to prove that $f(t, z)$ is holomorphic for $(t, z)$ in $B_{0} \times V$. Since each $f(t, z), t \in B_{0}$ is holomorphic for $z \in V$ and since $f(t, z)$ is uniformly bounded in $B_{0} \times V$ by (5.3), it thus suffices for $(a)$ to show that, for any fixed $z^{\prime} \in V$, it holds $\frac{\partial f\left(t, z^{\prime}\right)}{\partial \bar{t}}=0$ on $B_{0}$ in the sense of distribution; that is, it holds, for any $\varphi(t)=\varphi\left(t_{1}+i t_{2}\right) \in C_{0}^{\infty}\left(B_{0}\right)$,

$$
I:=\int_{B_{0}} f\left(t, z^{\prime}\right) \frac{\partial \varphi(t)}{\partial \bar{t}} d t_{1} d t_{2}=0 .
$$

To prove this by contradiction, assume that $I \neq 0$. We fix a small disk $V_{0}=\left\{\left|z-z^{\prime}\right|<r_{0}\right\} \Subset V$ of center $z^{\prime}$, so that we have $R_{n}^{\prime}(t) \ni V_{0}$ for any $t \in B_{0}$ and $n \geq \exists n_{0}$. We see from the mean-value theorem for holomorphic functions for $z$ that

$$
I=\frac{1}{\pi r_{0}^{2}} \iint_{B_{0} \times V_{0}} f(t, z) \frac{\partial \varphi(t)}{\partial \bar{t}} d t_{1} d t_{2} d x d y .
$$

We put $f_{n}(t, z)=\frac{\partial p_{n}(t, z)}{\partial z}$ in $B_{0} \times V$. Since $\lim _{n \rightarrow \infty} f_{n}(t, z)=f(t, z)$ uniformly on $V_{0}$ for a fixed $t \in B_{0}$ and since $f_{n}(t, z), f(t, z)$ are uniformly bounded in $B_{0} \times V_{0}$ by (5.3), the Lebesgue bounded theorem implies that

$$
I=\frac{1}{\pi r_{0}^{2}} \lim _{n \rightarrow \infty} \iint_{B_{0} \times V_{0}} f_{n}(t, z) \frac{\partial \varphi(t)}{\partial \bar{t}} d t_{1} d t_{2} d x d y .
$$


Therefore,

$$
\left|\frac{1}{\pi r_{0}^{2}} \iint_{B_{0} \times V_{0}} f_{n}(t, z) \frac{\partial \varphi(t)}{\partial \bar{t}} d t_{1} d t_{2} d x d y\right| \geq \frac{|I|}{2}>0 \quad \text { for } n \geq \exists N
$$

On the other hand, using Lemma 5.1(2)(ii) under Theorem 5.1(*), we see that, for a fixed $z \in V_{0}, p_{n}(t, z)$, and hence $f_{n}(t, z)$ is of class $C^{1}$ for $t \in B_{0}$. It follows that

$$
\int_{B_{0}} f_{n}(t, z) \frac{\partial \varphi(t)}{\partial \bar{t}} d t_{1} d t_{2}=-\int_{B_{0}} \varphi(t) \frac{\partial f_{n}(t, z)}{\partial \bar{t}} d t_{1} d t_{2}
$$

Hence, putting $I_{0}=\left(\pi r_{0}^{2}|I|\right) / 2>0$, we have from the Schwarz inequality that

$$
\begin{aligned}
I_{0}^{2} \leq & \left(\iint_{B_{0} \times V_{0}}|\varphi(t)|^{2} d t_{1} d t_{2} d x d y\right) \\
& \times\left(\iint_{B_{0} \times V_{0}}\left|\frac{\partial f_{n}(t, z)}{\partial \bar{t}}\right|^{2} d t_{1} d t_{2} d x d y\right) \\
= & : C \iint_{B_{0} \times V_{0}}\left|\frac{\partial f_{n}(t, z)}{\partial \bar{t}}\right|^{2} d t_{1} d t_{2} d x d y,
\end{aligned}
$$

where $C>0$ is independent of $n$. Lemma 4.1 and $\mathcal{L}^{\prime}(\mathrm{i})$ in "Preparation" for the pseudoconvex domain $\mathcal{R}_{n}^{\prime}$ imply that

$$
0 \leq \frac{4}{\pi} \int_{R_{n}^{\prime}(t)}\left|\frac{\partial f_{n}(t, z)}{\partial \bar{t}}\right|^{2} d x d y \leq \frac{\partial^{2} s_{n}(t)}{\partial t \partial \bar{t}} \quad \text { for any } t \in B \backslash \mathcal{L}^{\prime} .
$$

Since $\mathcal{L}^{\prime}$ (depending on $n$ ) consists of a finite number of $C^{\omega} \operatorname{arcs}$ in $B$, $R_{n}^{\prime}(t) \supset V_{0}$ for $n \geq n_{0}$, and $f_{n} \in C^{1}\left(B_{0} \times V_{0}\right)$, it follows that

$$
\begin{aligned}
I_{0}^{2} & \leq C \iint_{\left(B_{0} \backslash \mathcal{L}^{\prime}\right) \times V_{0}}\left|\frac{\partial f_{n}(t, z)}{\partial \bar{t}}\right|^{2} d t_{1} d t_{2} d x d y \\
& \leq \frac{C \pi}{4} \int_{B_{0} \backslash \mathcal{L}^{\prime}} \frac{\partial^{2} s_{n}(t)}{\partial t \partial \bar{t}} d t_{1} d t_{2} .
\end{aligned}
$$

We fix a disk $B_{1}: B_{0} \Subset B_{1} \Subset B$ and a $C_{0}^{\infty}$ function $\varphi_{1}(t) \geq 0$ on $B_{1}$ such that $\varphi_{1}(t) \equiv 1$ on $B_{0}$. Since $\frac{\partial^{2} s_{n}(t)}{\partial t \partial \bar{t}} \geq 0$ on $B_{1} \backslash \mathcal{L}^{\prime}$, we have that

$$
\int_{B_{0} \backslash \mathcal{L}^{\prime}} \frac{\partial^{2} s_{n}(t)}{\partial t \partial \bar{t}} d t_{1} d t_{2} \leq \int_{B_{1} \backslash \mathcal{L}^{\prime}} \varphi_{1}(t) \frac{\partial^{2} s_{n}(t)}{\partial t \partial \bar{t}} d t_{1} d t_{2} .
$$


Since $s_{n}(t)$ is of class $C^{1}$ on $B$ and $\varphi_{1}(t) \equiv 0$ on $\partial B_{1}$, we have that

$$
\int_{B_{1} \backslash \mathcal{L}^{\prime}} \varphi_{1}(t) \frac{\partial^{2} s_{n}(t)}{\partial t \partial \bar{t}} d t_{1} d t_{2}=\int_{B_{1}} s_{n}(t) \frac{\partial^{2} \varphi_{1}(t)}{\partial t \partial \bar{t}} d t_{1} d t_{2}
$$

both being equal to $-(1 / 4) \int_{B_{1}}\left(\frac{\partial \varphi_{1}}{\partial t_{1}} \frac{\partial s_{n}}{\partial t_{1}}+\frac{\partial \varphi_{1}}{\partial t_{2}} \frac{\partial s_{n}}{\partial t_{2}}\right) d t_{1} d t_{2}$. Therefore,

$$
\begin{aligned}
0<I_{0}^{2} & \leq \frac{C \pi}{4} \int_{B_{1}} s_{n}(t) \frac{\partial^{2} \varphi_{1}(t)}{\partial t \partial \bar{t}} d t_{1} d t_{2} \\
& \rightarrow \frac{C \pi}{4} \int_{B_{1}} s(t) \frac{\partial^{2} \varphi_{1}(t)}{\partial t \partial \bar{t}} d t_{1} d t_{2} \quad \text { as } n \rightarrow \infty \\
& =0 \quad \text { by the harmonicity of } s(t) \text { on } B
\end{aligned}
$$

which is a contradiction, and Step 1 is proved.

STEP 2. Assertion (2) is true.

In fact, fix $B_{i}, i=1,2, \ldots$, and let $P_{i}(t, z)$ denote the circular slit mapping for $(R(t), 0, \eta(t))$ used in $(a)$ in Step 1 for $\left.\mathcal{R}\right|_{B_{i}}$. From the theory of one complex variable, for a fixed $t \in B_{i} \cap B_{j}$, there exists $a_{i j}(t) \neq 0$ such that $P_{i}(t, z)=a_{i j}(t) P_{j}(t, z)$ on $R(t)$. Since $a_{i j}(t)$ is holomorphic on $B_{i} \cap B_{j}$ and since $\Delta$ is an open Riemann surface, we have a nonvanishing holomorphic function $a_{i}(t)$ on $B_{i}$ such that $a_{i j}(t)=a_{j}(t) / a_{i}(t)$ on $B_{i} \cap B_{j}$. Thus, $a_{i}(t) P_{i}(t, z)$ on $B_{i}, i=1,2, \ldots$ defines a holomorphic function $\mathcal{P}(t, z)$ on $\mathcal{R}$, so that $T:(t, z) \in \mathcal{R} \rightarrow(t, w)=(t, \mathcal{P}(t, z)) \in B \times \mathbb{P}_{w}$ proves Step 2 .

Corollary 5.1 (Rigidity). Let $\mathcal{R}: t \in \Delta \rightarrow R(t)$ satisfy (a)-(d). Assume that

(i) $R(t), t \in B$ satisfies Lemma 5.2( $(\star)$, so that $R(t)$ has $\nu$ (ideal) boundary components;

(ii) there exists at least one (ideal) boundary component $C(t)$ of $R(t), t \in \Delta$ such that $C(t)$ moves homotopically with $t \in \Delta$ in $\mathcal{R}$ and $C(t)$ is of positive harmonic measure on $R(t)$.

Let $\xi, \eta \in \Gamma(\Delta, \mathcal{R})$ such that $\xi \neq \eta$, and let $s(t), t \in \Delta$ denote the harmonic span for $(R(t), \xi(t), \eta(t))$. Then we have the following.

(1) In the case where $\Delta$ is an open Riemann surface, $s(t)$ is harmonic on $\Delta$ if and only if $\mathcal{R}$ is $\pi$-biholomorphic to a domain $(\Delta \times D) \backslash \Xi$ where $D$ is a circular $\mu$ slit domain in $\mathbb{P}_{w}$ and $\Xi:=t \in \Delta \rightarrow\left\{\xi_{k}(t)\right\}_{k=1, \ldots, \mu^{\prime}} \subset D$ is a multivalent holomorphic section of $\Delta \times D$ over $\Delta$, where $\mu \geq 1$ and $\mu+\mu^{\prime}=\nu$. Thus $s(t)$ is constant on $\Delta$. 
(2) In the case where $\Delta$ is a compact Riemann surface, then $\mathcal{R}$ is $\pi$ biholomorphic to the product $\Delta \times\left(D \backslash\left\{a_{k}\right\}_{=1, \ldots, \mu^{\prime}}\right)$, where $D$ is a circular $\mu$ slit domain in $\mathbb{P}_{w}$ and $a_{k} \in D$.

Proof. Since the proofs are similar, we prove (2). By (i), we cover $\Delta$ with disks $\left\{B_{i}\right\}_{i=1, \ldots, m}$ which satisfies Theorem $5.1(*)$, so that $s(t)$ is subharmonic on $B_{i}$ and on $\Delta$; hence, $s(t)=$ constant on $\Delta$. We fix $B_{i}$. Then by the proof of Theorem 5.1(2), the circular slit mapping $P_{i}(t, z)$ for $(R(t), 0, \eta(t))$ is holomorphic for $t \in B_{i}$. Since $D_{i}(t):=P_{i}(t, R(t))$ is a circular slit domain in $\mathbb{P}_{w}$ with $\nu$ circular arcs $\left\{A_{j}^{(1)}(t), A_{j}^{(2)}(t)\right\}$ (depending on $B_{i}$ ), some of which may be a point $A_{j}^{(1)}(t)=A_{j}^{(2)}(t)=: \xi_{j}(t)$, Behnke [3, p. 352] implies that each $A_{j}^{(k)}(t)$ is holomorphic on $B_{i}$. We rename $j$ such that $\operatorname{arc}\left\{A_{1}^{(1)}(t), A_{1}^{(2)}(t)\right\}=$ $P_{i}(t, C(t))$ for $C(t)$ in (ii); $\left\{A_{j}^{(1)}(t), A_{j}^{(2)}(t)\right\}, j=2, \ldots, \mu(\leq \nu)$, are arcs and the rest are points, say, $\xi_{k}(t), k=1, \ldots, \mu^{\prime}$. Under the homotopy condition for $C(t)$, we see by the same argument as in Theorem 4.1(3)(ii) that, if we put $\widetilde{P}_{i}(t, z):=P_{i}(t, z) / A_{1}^{(1)}(t)$ on $\mathcal{R}_{B_{i}}$ and $\widetilde{\xi}_{k}(t):=\xi_{k}(t) / A_{1}^{(1)}(t)$ on $B_{i}$, then $\widetilde{P}_{i}(t, z)=\widetilde{P}_{j}(t, z)$ on $\left.\mathcal{R}\right|_{B_{i} \cap B_{j}}$ for all $i, j$. We thus have a holomorphic function $\widetilde{P}(t, z)$ for $(t, z) \in \mathcal{R}$ such that $T:(t, z) \in \mathcal{R} \rightarrow(t, w)=(t, \widetilde{P}(t, z)) \in$ $\Delta \times \mathbb{P}_{w}$ is a $\pi$-biholomorphism from $\mathcal{R}$ onto $(\Delta \times D) \backslash \widetilde{\Xi}$, where $D$ is a circular $\mu$ slit domain in $\mathbb{P}_{w}$ and where $\widetilde{\Xi}=\left\{\widetilde{\xi}_{k}\right\}_{k=1, \ldots, \mu^{\prime}}$ is a $\mu^{\prime}$-valent holomorphic section of $\Delta \times D$ over $\Delta$. Taking the fundamental polynomials of $\left\{\widetilde{\xi}_{k}(t)\right\}_{k=1}^{\mu^{\prime}}$ on $\Delta$, we see that each $\widetilde{\xi}_{k}(t)$ is a constant $a_{k}$ on $\Delta$, which proves $(2)$.

Applying Corollary 5.1 to the special case $\left(\mathrm{c}^{\prime}\right)$ : each $R(t), t \in \Delta$ conformally equivalent to a disk $D$, we have the following.

Corollary 5.2. We have the following.

(1) Corollary 4.1 holds under the weaker condition for $\mathcal{R}: t \in \Delta \rightarrow R(t)$, which satisfies (a), (b), ( $\left.c^{\prime}\right)$, and (d).

(2) Let $\mathcal{R}: t \in \Delta \rightarrow R(t)$ satisfy (b), ( $\left.c^{\prime}\right)$, and (d), where $\Delta$ is a compact Riemann surface. Then, if there exist two distinct $\xi_{i} \in \Gamma(\Delta, \mathcal{R}), i=1,2$, then $\mathcal{R}$ is equivalent to the trivial $\Delta \times D$.

Acknowledgments. We thank M. Nakai for his helpful advice on harmonic spans and M. Brunella for his comment on Corollary 4.1. We also thank the referees for accurate comments, resulting in a revision of Section 2 and the addition of Remark 2.1. 


\section{REFERENCES}

[1] L.V. Ahlfors and L. Sario, Riemann Surfaces, Princeton Math. Ser. 26, Princeton University Press, Princeton, 1960.

[2] E. Bedford and B. Gaveau, Envelopes of holomorphy of certain 2-spheres in $\mathbb{C}^{2}$, Amer. J. Math. 105 (1983), 975-1009.

[3] H. Behnke, Die Kanten singuärer Mannigfaltigkeiten, Abh. Math. Semin. Univ. Hambg. 4 (1926), 347-365.

[4] M. Brunella, Subharmonic variation of the leafwise Poincaré metric, Invent. Math. 152 (2003), 119-148.

[5] L. Ford, Automorphic Functions, 2nd ed., Chelsea Publishing, New York, 1951.

[6] H. Grunsky, Neue Abschätzungen zur konformen Abbildung ein- und mehrfach zusammenhängender Beriche, Schr. Sem. Univ. Berlin 1 (1932), 95-140.

[7] R. Gunning and R. Narasimhan, Immersion of open Riemann surfaces, Math. Ann. 174 (1967), 103-108.

[8] S. Hamano, A lemma on $C^{1}$ subharmonicity of the harmonic spans for the discontinuously moving Riemann surfaces, preprint to appear in J. Math. Soc. Japan.

[9] - Variation formulas for $L_{1}$-principal functions and application to simultaneous uniformization problem, Michigan Math. J. 60 (2011), 271-288.

[10] - Variation formulas for principal functions, III: Applications to variation for Schiffer spans, preprint.

[11] N. Levenberg and H. Yamaguchi, The metric induced by the Robin function, Mem. Amer. Math. Soc. 448 (1991), 1-155.

[12] F. Maitani and H. Yamaguchi, Variation of Bergman metrics on Riemann surfaces, Math. Ann. 330 (2004), 477-489.

[13] M. Nakai and L. Sario, Classification Theory of Riemann Surfaces, Grundlehren Math. Wiss. 164, Springer, New York, 1970.

[14] Y. Nishimura, Immersion analytique d'une famille de surfaces de Riemann ouverts, Publ. Res. Inst. Math. Sci. 14 (1978), 643-654.

[15] T. Nishino, Function Theory in Several Complex Variables, Transl. Math. Monogr. 193, Amer. Math. Soc., Providence, 2001.

[16] M. Schiffer, The span of multiply connected domains, Duke Math. J. 10 (1943), 209216.

[17] H. Yamaguchi, Variations of pseudoconvex domains over $\mathbb{C}^{n}$, Michigan Math. J. 36 (1989), 415-457.

Sachiko Hamano

Department of Mathematics

Faculty of Human Development and Culture

Fukushima University

Fukushima 960-1296

Japan

hamano@educ.fukushima-u.ac.jp 
Fumio Maitani

2-7-7 Hiyoshidai

Ohtsu

Shiga 522-0112

Japan

hadleigh_bern@ybb.ne.jp

Hiroshi Yamaguchi

2-6-20-3 Shiromachi

Hikone

Shiga 522-0068

Japan

h. yamaguchi@s2.dion.ne.jp 